Research Article

\title{
Combination of Spectral Representation and Wavelet Packets for Generating Long-Period Ground Motions
}

\author{
Minghui Dai and Yingmin Li \\ College of Civil Engineering, Chongqing University, Chongqing 400045, China \\ Correspondence should be addressed to Yingmin Li; liyingmin@cqu.edu.cn
}

Received 24 November 2019; Revised 21 January 2020; Accepted 11 May 2020; Published 31 May 2020

Academic Editor: Pier Paolo Rossi

Copyright (C) 2020 Minghui Dai and Yingmin Li. This is an open access article distributed under the Creative Commons Attribution License, which permits unrestricted use, distribution, and reproduction in any medium, provided the original work is properly cited.

\begin{abstract}
Far-field long-period ground motions (hereafter long-period ground motions) featuring low-frequency components are responsible for the resonant responses of high-rise buildings. In this context, it is beneficial to assess the dynamic performance of these buildings under long-period ground motions with the aid of time history analysis. This paper proposes a method for generating long-period motions by combining long-period components synthesized by spectral representation with high-frequency components simulated by wavelet packets. Later-arriving long-period surface waves (LALP surface waves), which are determined on the grounds of phase dispersion, represent the main long-period properties in sense of velocity spectrum at longer periods of interest. An analytical expression for power spectrum density is employed to capture the narrowband properties of LALP velocity surface waves. Meanwhile, modification of the Gaussian random process is performed in time and frequency domains to attain a modulated initial seed motion, which shows the variability of the targeted ground motion. A simulation of high-frequency components is accomplished by means of iteration, in which wavelet coefficients of the modulated seed motion are adjusted to match the targeted response spectrum and cumulative energy plot. Furthermore, comparisons between an ensemble of realizations and target motions demonstrate the feasibility of the proposed method to generate long-period simulations sharing similar properties to target motions.
\end{abstract}

\section{Introduction}

Far-field long-period ground motions, which consist of dominant low-frequency components, are likely to enable high-rise buildings to excite resonance responses lasting several minutes [1]. It has been reported that damage to either structural components or non-structural components under long-period ground motions gives rise to unsafe occupancy and considerable maintenance expenses [2-5]. Thus, it is necessary to evaluate the performance of such structures subjected to long-period ground motions by means of time history analysis. However, networks with the capability to record a high signal-to-noise ratio over a longperiod range are insufficient. In addition, large-magnitude events in regions with active seismicity have low probabilities of occurrence, accounting for the low availability of long-period ground motions. In response to this challenge, artificial earthquake ground motions are essential to enrich electronic libraries of long-period ground motions.

The generation of ground motions compatible with a prescribed spectrum is attractive to engineers, since it is considered an extension of stochastic simulations. In essence, this process modifies the amplitudes of Fourier components in the frequency domain to attain a realization which closely matches a given response spectrum $[6,7]$. However, Fourier-based modification without involving time-varying frequency content fails to generate nonstationary of acceleration time series. To circumvent these disadvantages, wavelet analysis characterized by time-frequency discretization is introduced to generate artificial ground motions. For example, Spanos et al. [8,9] employed harmonic wavelets to synthesize artificial ground motions compatible with the design response spectra mandated by either Chinese or European aseismic regulation through 
iteratively modifying wavelet coefficients. As referred from the wavelet-packet-based stochastic model suggested by Yamamoto and Baker [10], Huang and Wang [11] accomplished the generation of artificial accelerograms conditional on agreement with the response spectrum in frequency domain and cumulative energy in time domain. However, a limitation of these methods is that the extent of compatibility with a target spectrum decreases as the periods shift towards a long-period range.

According to the seismological mechanism, long-period ground motions are characterized by LALP surface waves, which are converted from body waves at the margins of either basins or plains [12-15]. Because of these phenomena, the identification of long-period ground motions with broadband long-period characteristics is developed considering that energy from LALP components is predominantly relative to that of high frequencies [16]. However, it has been suggested that LALP surface waves in the form of velocity traces are equivalent to the summation of sinusoids with narrow-frequency components in special cases [17], as amplifications to the amplitudes of LALP surface waves occur at specific long periods. Thus, long-period ground motions share similar properties with pulse-like motions in view of long-period characteristics in frequency domain. Moustafa and Takewaki [18] adopted a function shaped-like Kronecker delta to describe frequency components of the pulse-like traces with one or few predominant frequencies on the basis of the stationary process [19-21]. In contrast, finite difference methods for long-period motions at the expense of computational resources and seismological information $[2,22,23]$ are not applicable for engineering communities. In this regard, the spectral representation for long-period components of long-period motions can be useful to satisfy demands in engineering practices.

The objective of this paper is to generate long-period ground motions through spectral representation for generating long-period components and wavelet packets for simulating high-frequency components. To achieve this aim, a time segment consisting of LALP components is truncated from acceleration time series on the basis of phase dispersion. Then, LALP surface velocity traces are simulated on the assumption of a stationary narrowband process. On the other hand, an initial seed motion is generated as a result of the modification of the Gaussian random process in time and frequency domains. Wavelet coefficients of the initial seed motion are adjusted by an iterative procedure to generate high-frequency components which are compatible with the target spectrum and cumulative energy plot. Finally, comparisons of simulations with target motions demonstrate that the proposed method has the potential to generate ground motions sharing similar long-period characteristics with target motions.

\section{Long-Period Components}

2.1. Determination of Long-Period Components. Similar to artificial generation for pulse-like ground motions $[20,24-26]$, determination of which fraction of a time history captures the gross long-period characteristics is a key issue in the separation of surface waves from body waves. This facilitates subsequent numerical modelling. Actually, there is no principle to conduct separation of long-period surface waves from its time history as accurately as possible, since the components which are dependent on frequencies always overlap in most cases. However, with reference to articles [14, 15], variations in the speed of wave trains in propagation media account for phase dispersion, which are referred to as "envelope delays" [13]. Specifically, the speeds of long-period surface waves increase with periods, as opposed to those of body waves, which decrease with periods.

To illustrate phase dispersion in nature, we estimate the arrival times of phases which are dependent on periods by means of an array of narrowband Gaussian filters [27, 28]. Two acceleration time histories for CHB002EW and TKY023EW with epicentral distances $367 \mathrm{~km}$ and $377 \mathrm{~km}$ during the 2011 Tohuku, M9 earthquake, are considered representatives of long-period ground motions. Thus, we use them as examples to illustrate the proposed method in the following sections. As shown in Figure 1, phases with periods of 2, 5, 7, and $10 \mathrm{sec}$ are individually extracted from the two records by using Gaussian filters. The variations of phases are shown in the form of arrival times corresponding to envelope peaks. It is seen that the arrival times of the longperiod phases tend to arrive later with periods decreasing, while the $2 \mathrm{sec}$ phase arrives at nearly the same time as that for PGA. This general trend coincides with the results from the analysis of long-period motions in the Po Plain of Italy [27].

The principle of determining long-period components is defined herein. It is conditioned that the truncated time series should match well with its original record in terms of long-period characteristics. Following this line, the steps are as follows.

First, the maximum interval $t_{\mathrm{mc}}$ of zero-level crossings for acceleration time series is calculated such that the period $\left(p=2 t_{\mathrm{mc}}\right)$ of the earliest-arrival long-period component is determined based on phase dispersion illustrated above; second, the phase with the period of $p$ is extracted from its acceleration time series by using the designed narrowband Gaussian filter; finally, a reference time point $t_{l}$ which is used to determine the LALP surface waves should be satisfied with the condition, $t_{l} \in\left(t_{p g a}, t_{p}\right]$. At this point, we could possibly search for an appropriate $t_{l}$, after which the time segment consisting of sufficient surface waves enables the velocity response spectrum of the time segment to show an agreement with that of its original record around dominant long periods. Note that $t_{p}$ is the first wave pack of the filtered phase shown in Figure 2 and $t_{p g a}$ is the arrival time for PGA.

The detailed explanation of the condition for $t_{l}$ is that the $t_{p}$ is often treated as an arrival time for the filtered phase with a period of $p[15,27]$; however, due to phase dispersion and scatting effects, it is expected that the truncated time segment contains sufficient surface waves energy at the dominant period as much as possible, thus resulting in the upper bound for $t_{p}$; meanwhile, it is reasonable that laterarriving surface waves are converted from body waves $[14,15]$, thus resulting in the lower bound for $t_{p g a}$. 

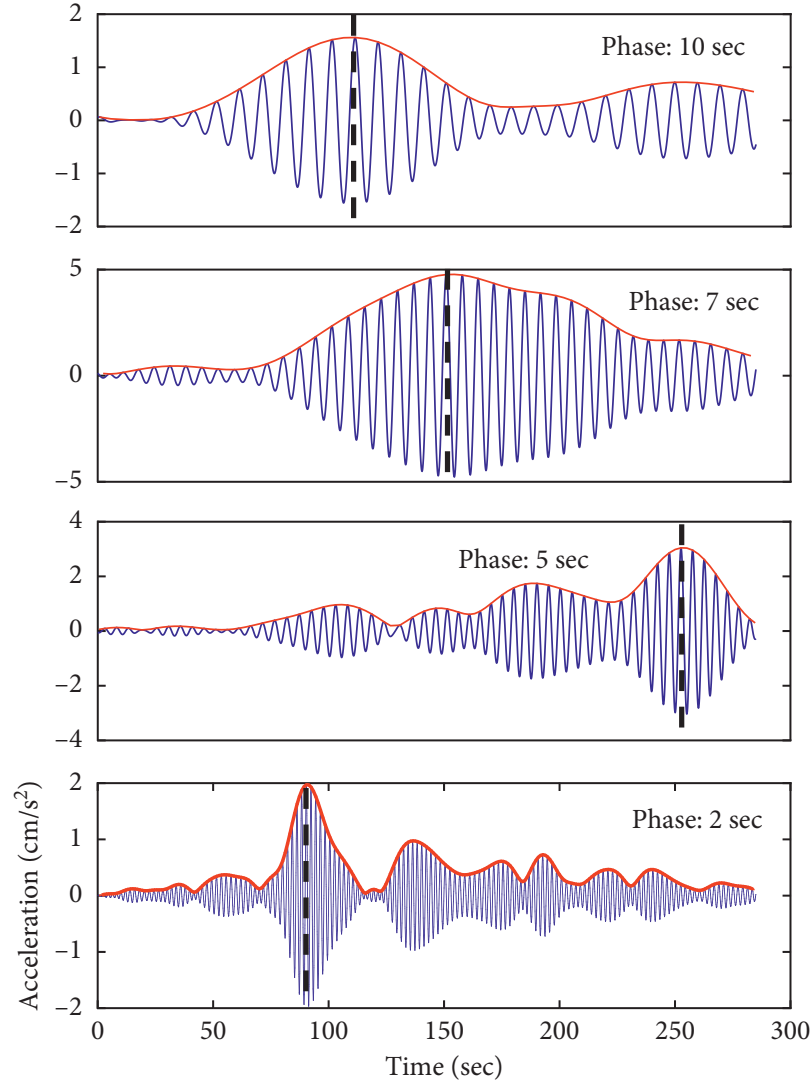

(a)
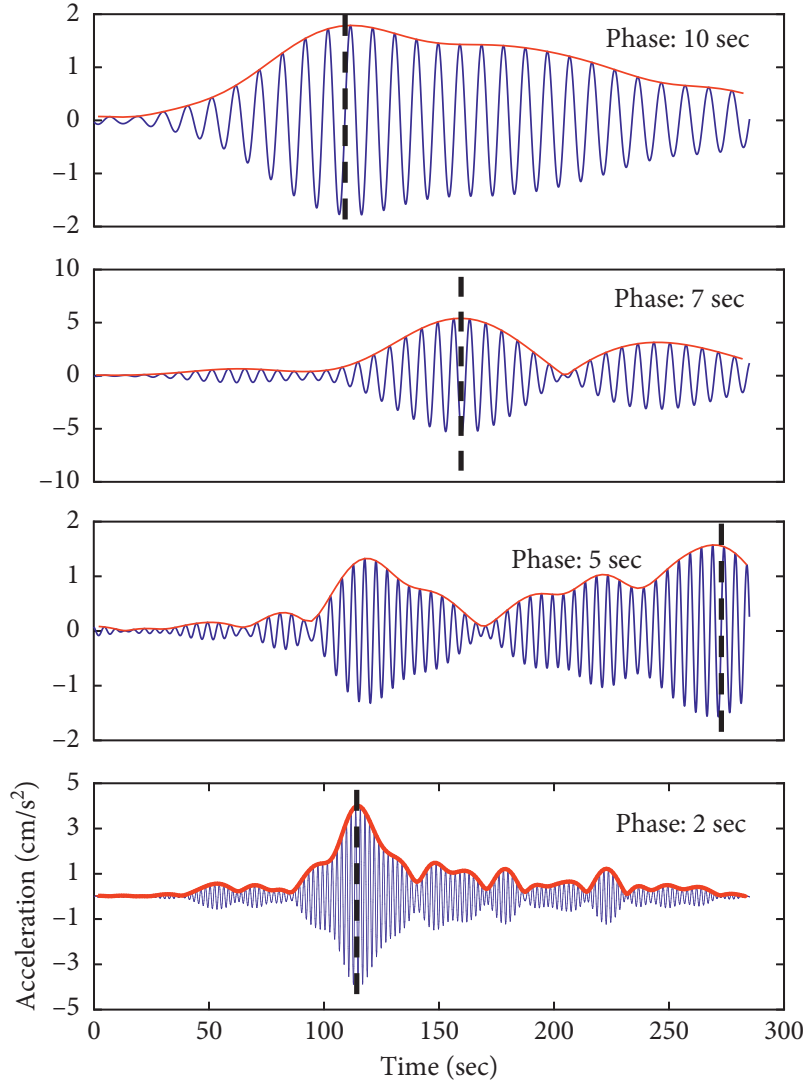

(b)

FIgURe 1: Phases dependent on periods from 2, 5, 7, and $10 \mathrm{sec}$ are attained from the acceleration traces of (a) CHB002EW and (b) TKY023EW, by using a set of Gaussian filters.

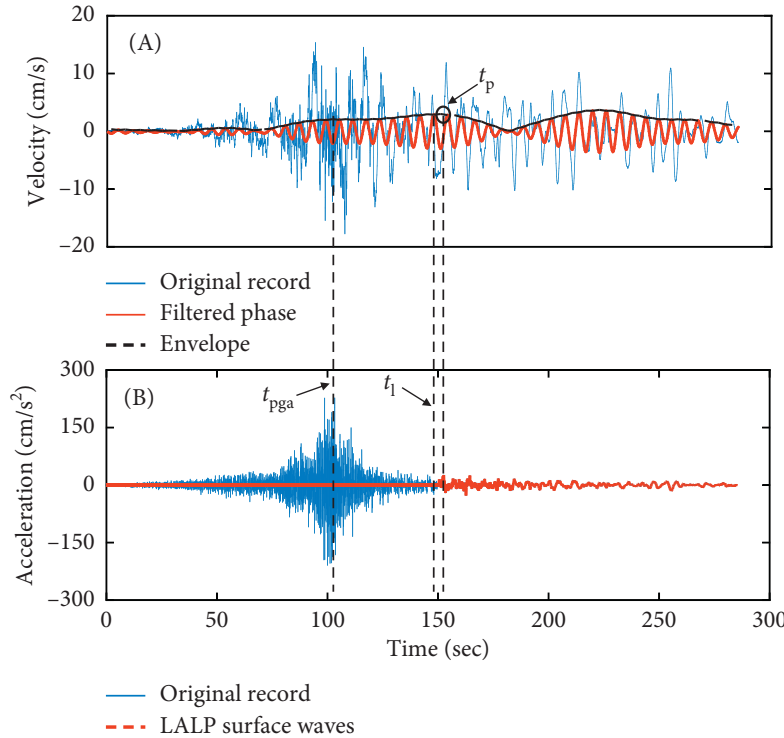

(a)

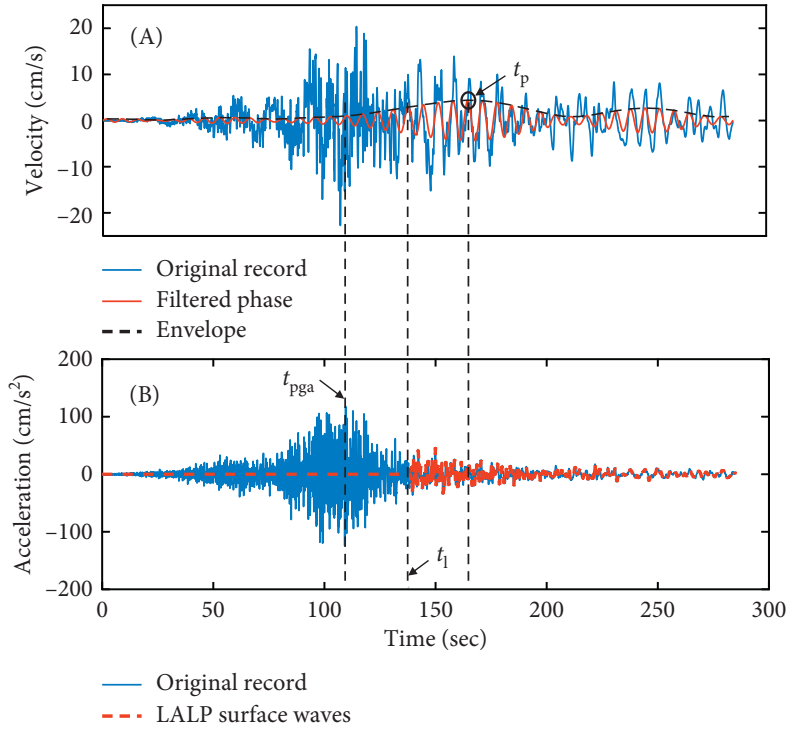

(b)

Figure 2: Illustration of determination of long-period components for (a) CHB002EW and (b) TKY023EW.

As a result, the examples are shown in Figure 2. The $t_{\mathrm{mc}}$ for CHB002EW is $3.04 \mathrm{sec}$, and the corresponding $p=2 \times 3.04=6.08 \mathrm{sec}$; a Gaussian filter with a centre period of $p$ is employed to extract the phase shown in Figure 2(a); then, as shown in Figure 2(b), a time segment mainly consisting of long-period surface waves is truncated from its 
acceleration time series with reference to the $t_{l}$, and this is conditioned by its velocity spectrum matching well with that of the original record (illustrated in Figure 3). Similarly, the process for TKY023EW is illustrated in Figure 2(b). The identified parameters of $t_{l}$ for these records are listed in Table 1.

It is observed from Figure 3 that the velocity response spectra of the truncated waves at long predominant periods show an agreement with those of the corresponding original records. In contrast, there are obvious deviations at short periods. In this regard, the truncated waves are capable of capturing the gross long-period characteristics of the original records.

2.2. Representation of Long-Period Components. As illustrated above, truncated waves with gross long-period properties are characterized by narrowband properties. Because of this, the simulation of long-period components consisting of LALP surface waves is generated on the basis of the stationary power spectrum density function (PSDF). Accordingly, an exponential form for modelling the PSDF of LALP surface waves is written as in equation (1), as is derived from the works regarding long-period waves:

$$
S(\omega)=\frac{\alpha}{\omega^{p}} \exp \left[-\beta \frac{1}{\omega^{q}}\right],
$$

where $\alpha$ is the scaling constant to modulate the amplitudes; $\omega$ denotes the angular frequency $(\mathrm{rad} / \mathrm{s}) ; \beta, p$, and $q$ are coefficients related to the frequency domain.

The derivative of the form with respect to angular frequency $\omega$ is given as follows:

$$
\frac{\mathrm{d} s(\omega)}{\mathrm{d} \omega}=\frac{\alpha(-p)}{\omega^{p+1}} \exp \left(-\frac{\beta}{\omega^{q}}\right)+\frac{\alpha}{\omega^{p}} \exp \left(-\frac{\beta}{\omega^{q}}\right)(-\beta)\left(\frac{-q}{\omega^{q+1}}\right) .
$$

It is easy to know that the maximum value for $s(\omega)$ is attained when $\mathrm{d} s(\omega) / \mathrm{d} \omega$ equals zero, and the predominant angular frequency is determined. Then, equation (2) can be rewritten as

$$
\frac{\alpha p}{\omega^{p+1}} \exp \left(-\frac{\beta}{\omega^{q}}\right)=\frac{\alpha p}{\omega^{p}} \exp \left(-\frac{\beta}{\omega^{q}}\right)\left(\frac{\beta q}{\omega^{q+1}}\right) .
$$

Solving this equation, the predominant frequency is shown as follows:

$$
f_{d}=\frac{1^{q}}{2 \pi} \sqrt{\frac{\beta q}{p}}
$$

For equation (4), coefficients $\beta, p$, and $q$ collectively control the predominant frequency, and the variability of the PSDF is available by modulating the relative proportion among three coefficients. As shown in Figure 4, the normalized PSDs with various parameters of $\beta, p$, and $q$ demonstrate the ability of the function to capture narrow bandwidth where the greatest portion of energy is concentrated. At the same time, it is found that the bandwidth

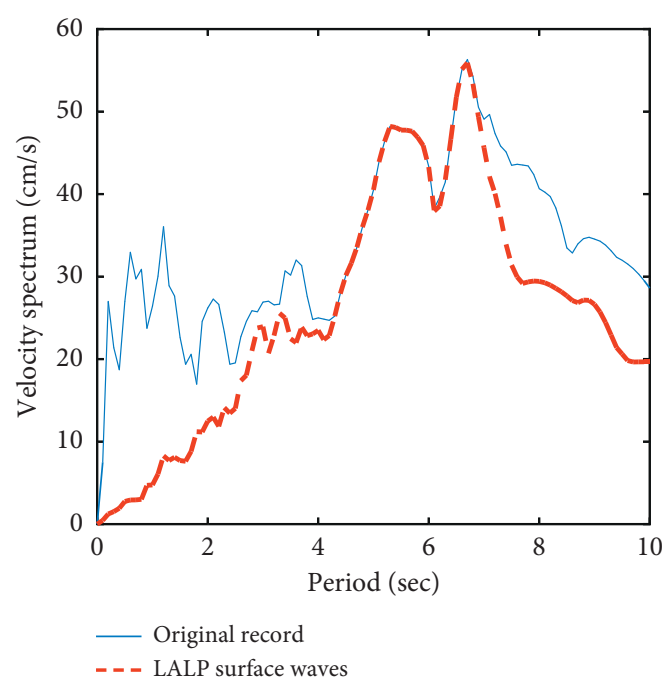

(a)

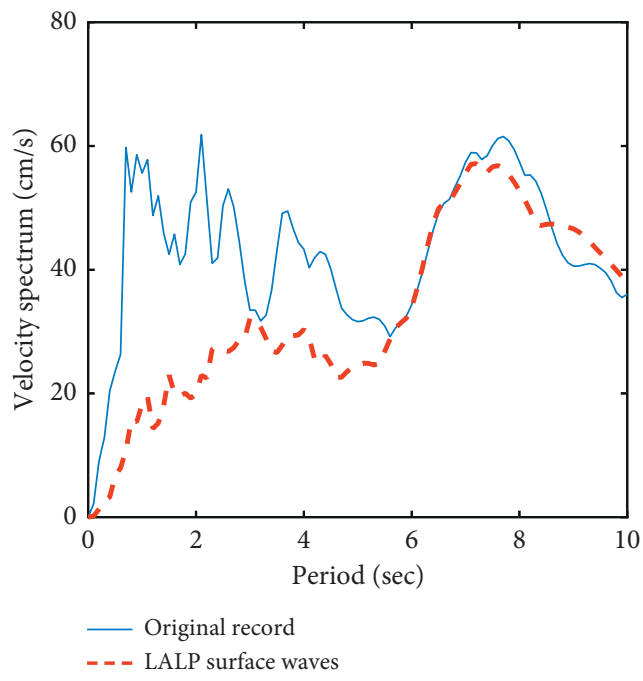

(b)

FIgURE 3: Comparison of truncated time series with corresponding original records in terms of velocity response spectrum. (a) CHB002EW and (b) TKY023EW.

TABLE 1: Parameters of determination and PSD for truncated longperiod waves.

\begin{tabular}{lccccc}
\hline Record & $t_{l}(\mathrm{sec})$ & $\alpha$ & $\beta$ & $p$ & $q$ \\
\hline CHB002EW & 150.58 & 275.41 & 0.67 & 18.14 & 11.47 \\
TKY023EW & 138.86 & 107.07 & 0.35 & 12.01 & 9.96 \\
SIT003EW & 157.33 & 9999.03 & 4.62 & 8.96 & 3.59 \\
\hline
\end{tabular}

becomes wider when the predominant frequency shifts towards higher frequencies.

An actual PSD is estimated from LALP velocity surface waves using the modified periodogram of the Welch technique with Gaussian smoothing. Identification of the parameters is performed by fitting the analytical function equation (1) to the actual PSD in a nonlinear least-square sense. Thus, the fitting formula is presented as follows: 


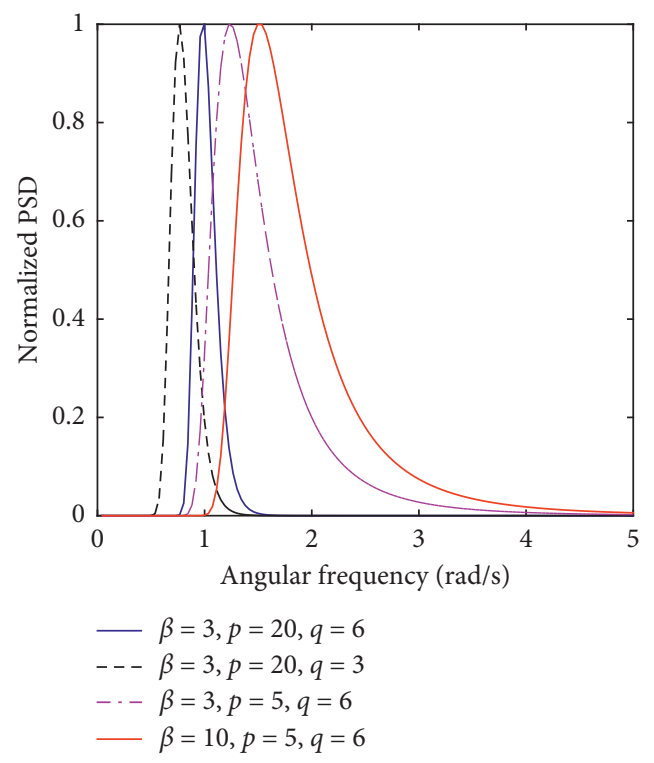

FIGURE 4: Normalized power spectrum density with various parameters of $\beta, \rho$, and $q$.

$$
[\widehat{\alpha}, \widehat{\beta}, \widehat{p}, \widehat{q}]=\underset{\alpha, \beta, p, q}{\arg \min } \sum_{i=1}^{n}[\operatorname{PSD}(\omega)-s(\omega ; \alpha, \beta, p, q)]^{2} .
$$

As a consequence, the parameters of $\alpha, \beta, p$, and $q$ for the records CHB002EW and TKY023EW are tabulated in Table 1. Comparisons of the resulting PSDs with the actual PSDs are illustrated in Figures 5(a) and 5(b), respectively. In general, it is clear that the analytical functions perform well in modelling actual PSDs considering the narrowband properties.

For the temporal domain, we use Hilbert transform to obtain instantaneous amplitudes of the velocity time series. The envelope $e_{l}(t)$ for LALP velocity surface waves in time series is calculated as follows:

$$
e_{l}(t)=\sqrt{v(t)^{2}+H[v(t)]^{2}},
$$

in which $H[v(t)]$ is the Hilbert transform, $v(t)$ is the velocity time series, and $t$ is the time instant.

As the instantaneous amplitudes are shown in Figure 6, the multiple local maxima increase the difficulty in selecting an appropriate envelope model. To reduce deviations, the third level envelope of LALP velocity surface waves is attained by using the multimodal point method [29]. Then, the double-exponent function in the following equation is used to reflect the change in amplitudes with time:

$$
\bar{e}_{l}(t)=c_{1} \exp \left(\lambda_{1} t\right)+c_{2} \exp \left(\lambda_{2} t\right)
$$

where $c_{1}, c_{2}, \lambda_{1}$, and $\lambda_{2}$ are the fitted coefficients and $\bar{e}_{l}$ denotes the fitted envelope.

Correspondingly, the fitted envelopes $\bar{e}_{l}$ presented in Figure 6 are attained as a result of the fitting process, and fitted parameters are listed in Table 2.
It is observed that the fitted envelopes $\bar{e}_{l}$ are capable of capturing the trend of amplitudes with time evolution, and thus, the function can adapt to the different shapes by modulating the parameters.

Considering the characteristics of PSD for LALP velocity time series, the time series is treated as a summation of cosine waves with narrowband frequencies in the sense of spectral representation, as defined in the following equation:

$$
x(t)=2 \bar{e}_{l} \sum_{n=1}^{N}[s(\omega ; \alpha, \beta, p, q) 2 \pi \Delta f]^{1 / 2} \cos \left(2 \pi f_{n}+\phi_{n}\right),
$$

where $\bar{e}_{l}$ is the fitted envelope of the LALP velocity time series; $s(\omega ; \alpha, \beta, p, q)$ denotes the power spectral density function with parameters of $\alpha, \beta, p, q ; f_{n} \in\left[f_{l}, f_{u}\right]$ is the frequency band; $f_{l}$ and $f_{u}$ are the lower and upper cutoff frequencies of the PSDF, respectively; the frequency step is $\Delta f=\left(f_{u}-f_{l}\right) / N ; f_{u}$ is dependent on sampling rate of the time series; and $f_{l}$ is $0.01 \mathrm{~Hz}$; each discrete frequency is $f_{n}=f_{l}+n \Delta f ; \varnothing_{n}$ is a set of random phase angles distributed uniformly over the range of $[0,2 \pi]$; and $N$ is the number of time steps.

Note that the acceleration time history should be performed in baseline correction through a fourth-order Butterworth filter with bandwidth ranging from 0.1 to $1 \mathrm{~Hz}$. This enables the removal of spurious drifts and the retention of the long-period properties. Consequently, it is seen from Figure 7 that the realizations present stochastic properties in time domain, but predominant frequencies controlling the LALP surface waves cannot vary over time.

Comparisons of the simulations with truncated longperiod surface waves in terms of $5 \%$ damping spectra are shown in Figure 8. It is found that the simulated spectra share a similar predominant period with the truncated 


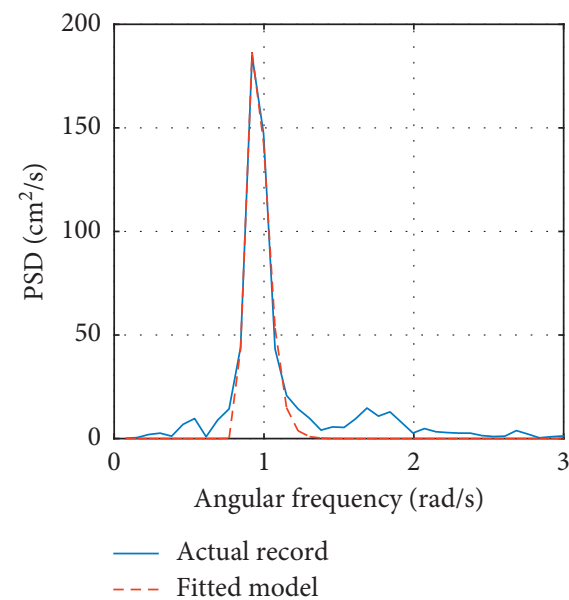

(a)

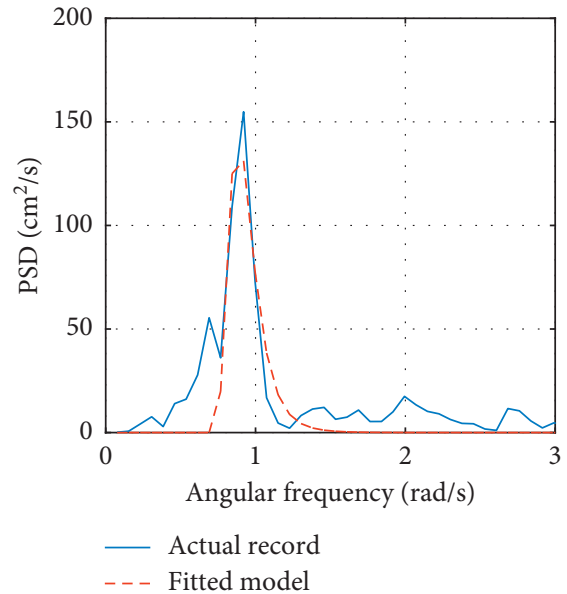

(b)

FIGURE 5: PSD for truncated LALP velocity time series. (a) CHB002EW and (b) TKY023EW.

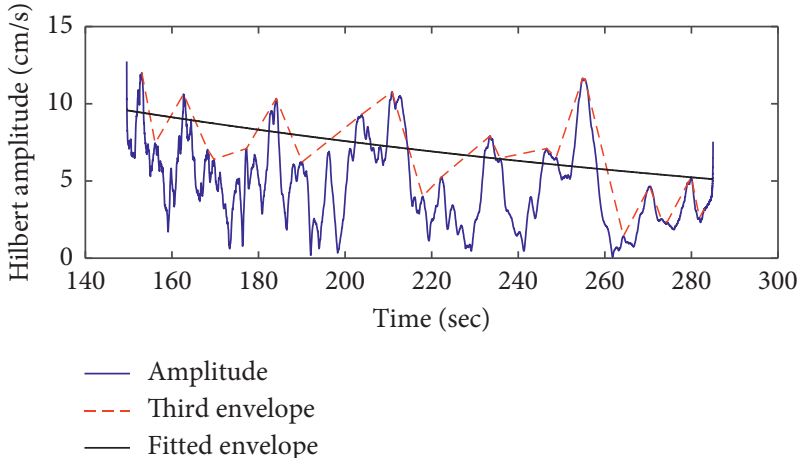

(a)

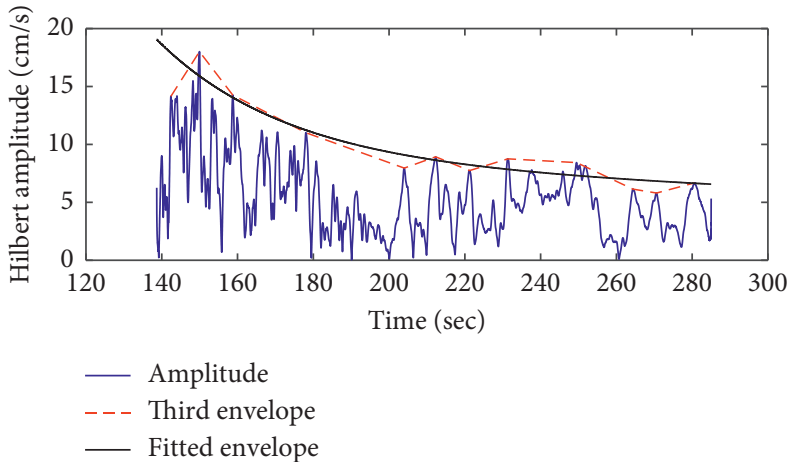

(b)

FIGURE 6: Instantaneous amplitudes for truncated velocity time series. (a) CHB002EW and (b) TKY023EW.

TABLE 2: Envelope parameters for truncated long-period velocity surface waves.

\begin{tabular}{lcccc}
\hline Record & $c_{1}$ & $\lambda_{1}$ & $c_{2}$ & $\lambda_{2}$ \\
\hline CHB002EW & 5.83 & -0.0031 & 3.21 & -0.0030 \\
TKY023EW & 12.80 & -0.11 & 12.90 & -0.0062 \\
SIT003EW & 13.88 & -0.011 & 10.55 & -0.011 \\
\hline
\end{tabular}

waves. This is consistent with the results in Figure 5. Meanwhile, the truncated waves almost fluctuate between the median plus or minus one standard deviation $\pm \sigma$. Because of this, the truncated waves are possibly treated as one sample of the realizations on the basis of stochastic properties.

\section{Wavelet Packets}

Wavelet packets analysis is applied to the decomposition of a time series $x(t)$ into a set of wavelet coefficients $c_{j, k}^{i}$. This means that energy is localized in both time and frequency domains. The basic expression in the form of convolution is defined as follows:

$$
c_{j, k}^{i}=\int_{-\infty}^{\infty} x(t) \psi_{j, k}^{i}(t) \mathrm{d} t
$$

where $\psi_{j, k}^{i}(t)$ is the $i^{\text {th }}$ set of the modified mother wavelet, which is determined by both the $j^{\text {th }}$ scale parameter that dilates in frequency domain and the $k^{\text {th }}$ translation parameter that shifts through the time axis. The Meyer wavelet is chosen as the mother wavelet herein, as proposed in similar work [10].

Conversely, a time series $x(t)$ of $2^{N}$ reconstructed from the wavelet coefficients on the basis of inverse wavelet packets transform is given as follows:

$$
x(t)=\sum_{i=1}^{2^{j}} \sum_{k=1}^{2^{N-j}} c_{j, k}^{i} \psi_{j, k}^{i}(t) .
$$

On the other hand, the trade-off between frequency and time resolution should be taken into account because the resolution is in accordance with the Heisenberg uncertainty principle. This means that the high resolution of frequency 

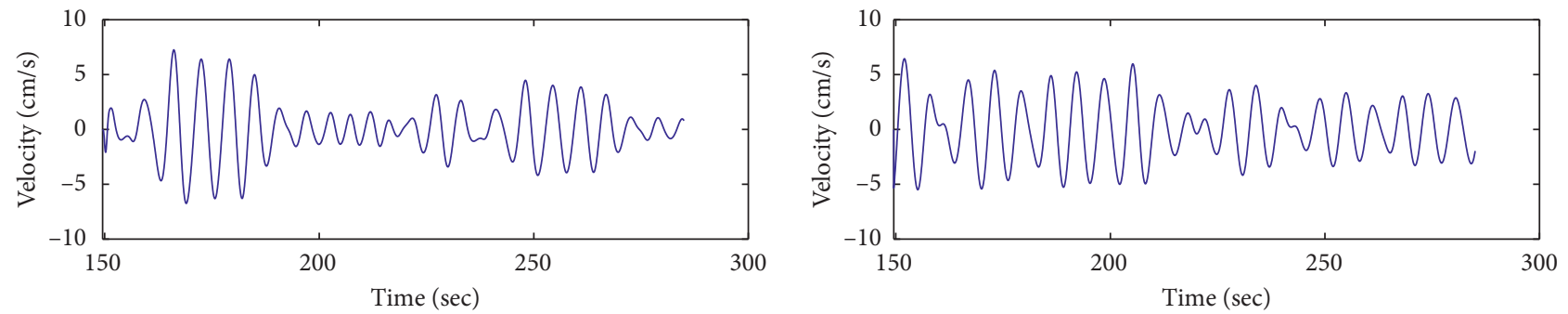

(a)
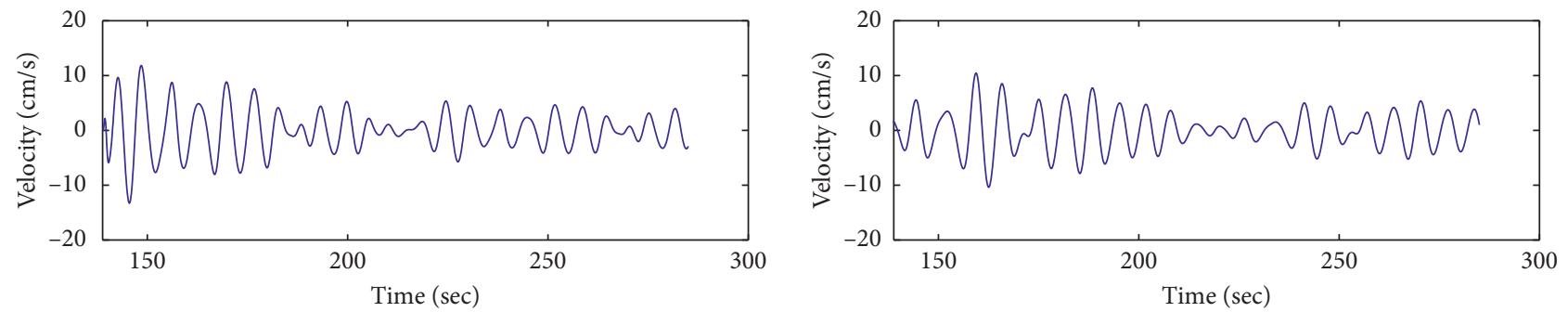

(b)

FIGURE 7: Simulated long-period surface waves in the form of velocity time series. (a) CHB002EW and (b) TKY023EW.

information comes at the expense of low resolution in temporal domain. Accordingly, the time resolution $\mathrm{d} t_{w}$ and frequency resolution $\mathrm{d} f_{w}$ are expressed in equations (11) and (12), respectively, after the $j^{\text {th }}$ decomposition step. The product of $\mathrm{d} t_{w}$ and $\mathrm{d} f_{w}$ is a constant in equation (13):

$$
\begin{aligned}
\mathrm{d} t_{w} & =2^{j} \mathrm{~d} t, \\
\mathrm{~d} f_{w} & =\frac{f_{N}}{2^{j}}, \\
\mathrm{~d} t_{w} \cdot \mathrm{d} f_{w} & =\frac{1}{2},
\end{aligned}
$$

where $j$ is the scale parameter, $\mathrm{d} t$ is the time interval of a time series, and $f_{N}$ is the Nyquist frequency determined by the sampling rate of the time series. Compared with the time resolution, detailed information related to frequency domain is of interest in engineering practice [11]. This facilitates simulations in sense of compatibility with a target spectrum.

To illustrate above, the time history for $\mathrm{CHB} 002 \mathrm{EW}$ with the sampling interval $\mathrm{d} t=0.01$ is decomposed into 512 frequency rows at the $9^{\text {th }}$ decomposition depth. Correspondingly, the frequency interval descends to $0.0976 \mathrm{~Hz}$, while the corresponding time interval between the centres of adjacent wavelet packets enlarges to $\mathrm{d} t_{w}=2^{9} * 0.01=5.12 \mathrm{sec}$.

As a result, Figure 9 illustrates the squared wavelet coefficients $\left|c_{j, k}^{i}\right|^{2}$ related to the time-frequency distribution. For frequency domain, the majority of coefficients at frequencies ranging from 4 to $7 \mathrm{~Hz}$ correspond to the peak values of the acceleration response spectrum in Figure 9(c); for temporal domain, the majority of coefficients occurring at approximately $100 \mathrm{sec}$ correspond to a strong shocking phase during which the cumulative energy curve shows a sharp increase in Figure 9(d). This suggests that the wavelet packets transform has the potential to capture the characteristics pertaining to intensity and spectrum, each of which is well correlated with the time and frequency domains, respectively.

However, it should be kept in mind that the capability of wavelet packets to detect the components at longer periods cannot be the same as that for high-frequency components, because of shifting and scaling based on a power of 2 . To illustrate this, the resulting frequency interval $f \in[0.0977,0.1953]$ leads to a jump from 5.12 to $10.23 \mathrm{sec}$ in the form of period. This means that wavelet analysis is not suitable for discerning long-period components in frequency domain. Instead, it specializes in localization of energy at high frequencies.

At this point, high-frequency components are generated for the purpose of compatibility with a given spectrum in frequency domain and cumulative energy in time domain, as referred from the method proposed by Huang and Wang [11].

\section{Formation of High-Frequency Components Using Wavelet Packets}

4.1. Generation of Initial Seed Motion. Prior to the generation of high-frequency components, an initial seed motion should be created, because variability of the initial seed motion could influence nonstationary properties of desired motions $[11,30]$. Thus, the initial seed motions for highfrequency components are created as a result of the modulation of the Gaussian random process with respect to time and frequency domains.

4.1.1. Modulation in Time Domain. By visual observation of the acceleration waveforms shown in Figure 2, a piecewise function is employed to modulate the Gaussian random 

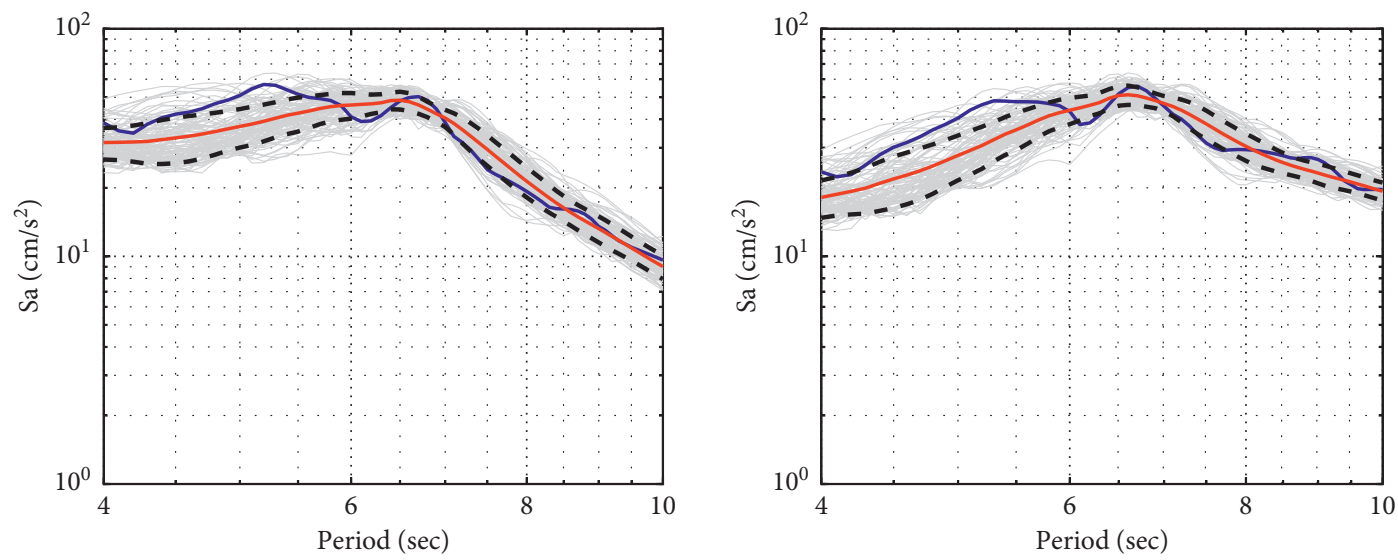

CHB002EW

_ Truncated long-period surface waves

_ Simulated

— Simulations: median

- - - Simulations: median $+/-1 \sigma$

CHB002EW

_ Truncated long-period surface waves

_ Simulated

- Simulations: median

- - - Simulations: median $+/-1 \sigma$

(a)
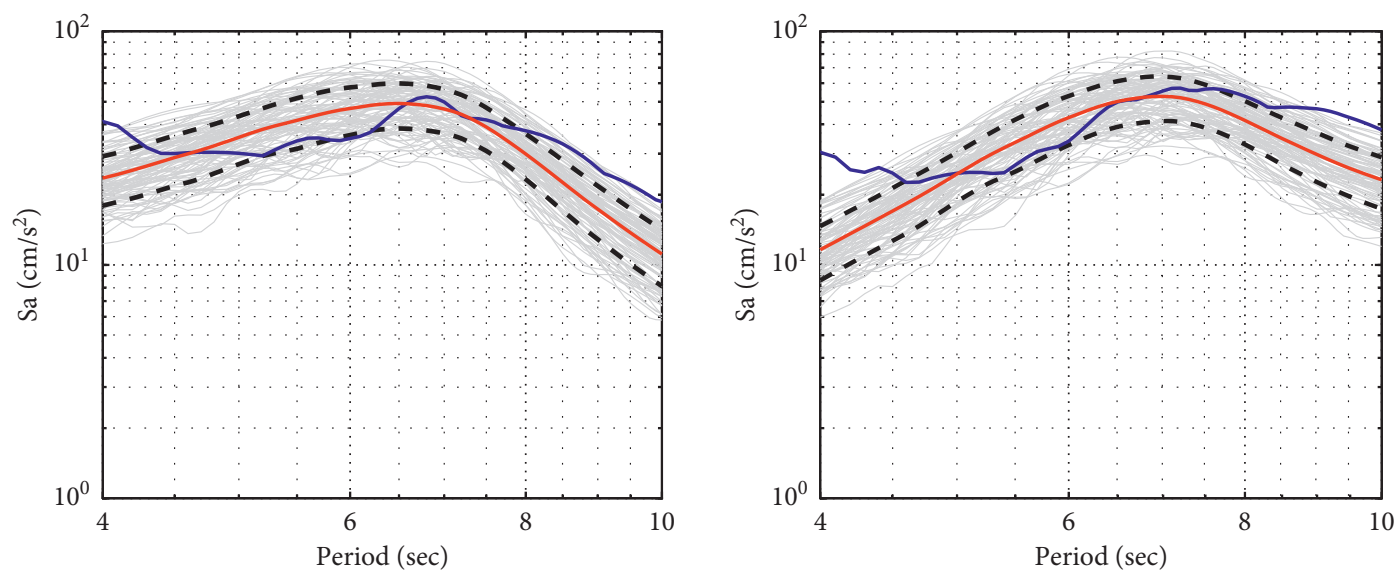

TKY023EW

_ Truncated long-period surface waves

_ Simulated

— Simulations: median

- - Simulations: median $+/-1 \sigma$

TKY023EW

_ Truncated long-period surface waves

_ Simulated

— Simulations: median

- - Simulations: median $+/-1 \sigma$

(b)

FIGURE 8: Response spectra for an ensemble of realizations of truncated long-period surface waves from two records of CHB002EW and TKY023EW (solid blue line). Of 100 simulated ground motions (thin gray lines), their median (thick red line) and median plus and minus one standard deviation $\pm \sigma$ (dashed lines) are involved; acceleration spectra on the left side; velocity spectra on the right side. (a) CHB002EW and (b) TKY023EW.

process in time domain, and its mathematical expression is written as follows:

$$
e_{h}(t)= \begin{cases}0, & t \leq t_{0}, \\ \left(\frac{t-t_{0}}{t_{m}-t_{0}}\right)^{\gamma}, & t_{0}<t \leq t_{m}, \\ \exp ^{-\eta\left(t-t_{m}\right)}, & t>t_{m},\end{cases}
$$

where $t_{m}$ denotes the time corresponding to PGA; $\gamma$ and $\eta$ are parameters controlling the ascent and descent stage of the time segment before and after $t_{m}$, respectively; $t_{0}$ indicates the onset of a ground motion. Correspondingly, as shown in Figure 10, a set of normalized envelopes regarding various parameters of $t_{m}, \gamma$, and $\eta$ demonstrates the variability of waveform shapes.

Furthermore, a normalized cumulative energy model available from integration of the piecewise function with respect to time is given as follows: 


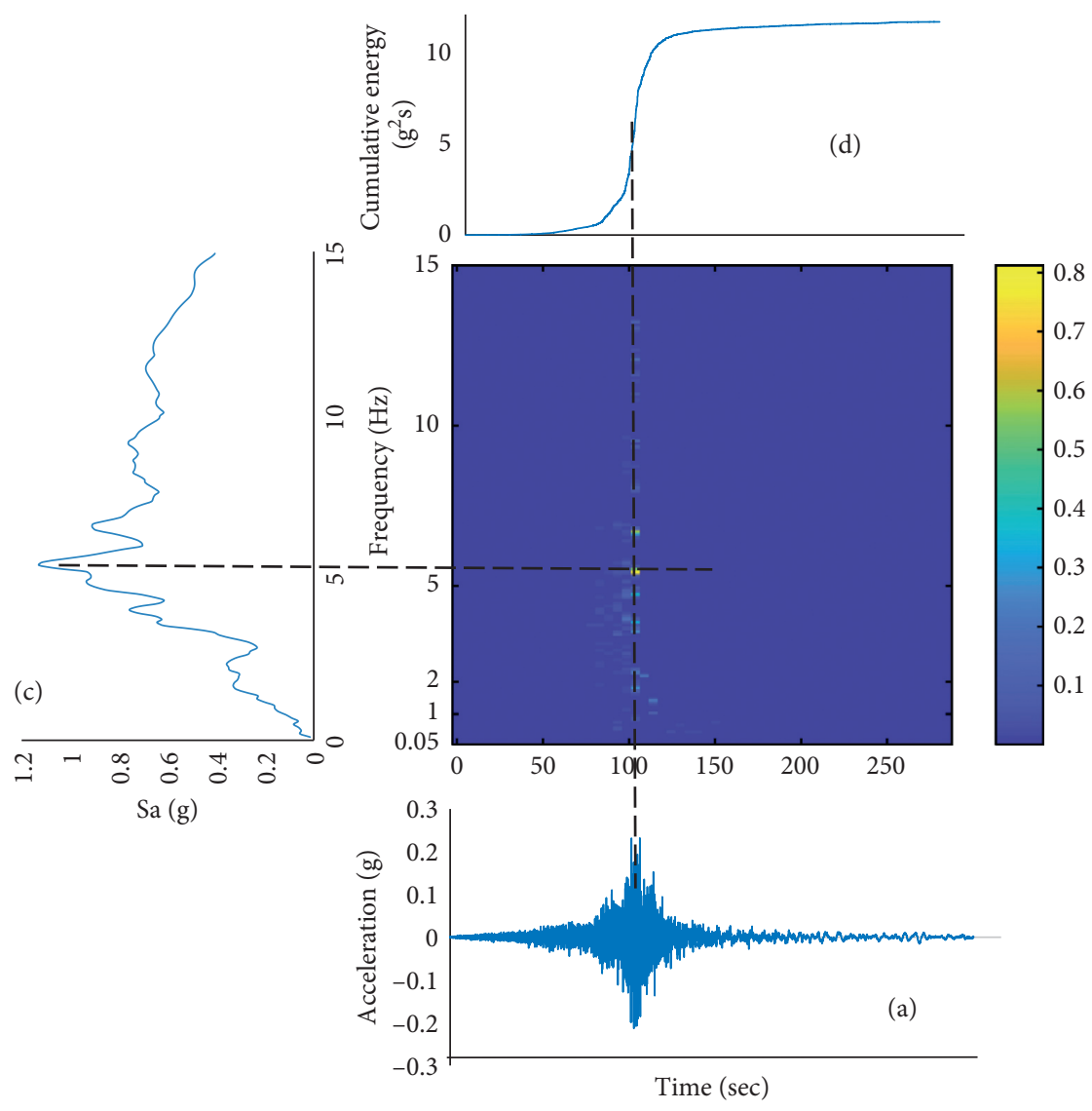

(b)

FIGURE 9: Relationships pertaining to time and frequency domains are illustrated through wavelet packets decomposition for the record CHB002EW: (a) acceleration time history; (b) distribution of squared wavelet coefficients; (c) acceleration response spectrum; (d) cumulative energy curve.

$$
h(t)=\frac{\int e_{h}^{2}(t) \mathrm{d} t}{\int_{0}^{T} e_{h}^{2}(t)^{2} \mathrm{~d} t}=\frac{1}{\left(2 \eta+\left(\left(t_{m}-t_{0}\right) /(2 \gamma+1)\right)\right)} \begin{cases}0, & t \leq t_{0}, \\ \left(\frac{t_{m}-t_{0}}{2 \gamma+1}\right)\left(\frac{t-t_{0}}{t_{m}-t_{0}}\right)^{2 \gamma+1}, & t_{0}<t \leq t_{m}, \\ -\frac{1}{2 \eta} \exp ^{-2 \eta\left(t-t_{m}\right)}+\frac{1}{2 \eta}+\left(\frac{t_{m}-t_{0}}{2 \gamma+1}\right), & t>t_{m} .\end{cases}
$$

Thus, the parameters of the envelope function are attained by minimizing the integrated differences between the normalized actual energy curve and piecewise energy model, that is,

$$
\begin{aligned}
{\left[\widehat{t}_{0}, \widehat{\gamma}, \widehat{\eta}\right] } & =\underset{t_{0}, \gamma, \eta}{\arg \min } \int_{0}^{t}\left[\int_{0}^{t} h^{2}(t) \mathrm{d} t-\int_{0}^{t} h_{a}^{2}(t) \mathrm{d} t\right]^{2} \mathrm{~d} t \\
h_{a}(t) & =\frac{\int_{0}^{t} x^{2}(t) \mathrm{d} t}{\int_{0}^{D} x^{2}(t) \mathrm{d} t},
\end{aligned}
$$

where $x(t)$ is the acceleration time series, $D$ is the duration of an acceleration time series, and $h_{a}(t)$ denotes the normalized cumulative energy. The fitted parameters are listed in Table 3.

It is found from Figure 11 that the fitted curves are in close agreement with their actual curves at the strong shaking phases, during which the curves appear to be a vertical line. Meanwhile, discrepancies occur at approximately $140 \mathrm{sec}$, after which LALP surface waves arrive. Although the amplitudes of LALP surface waves are relatively small, in distinct contrast to those of body waves, they have a low attenuation rate. This results from the longperiod properties of surface waves. 


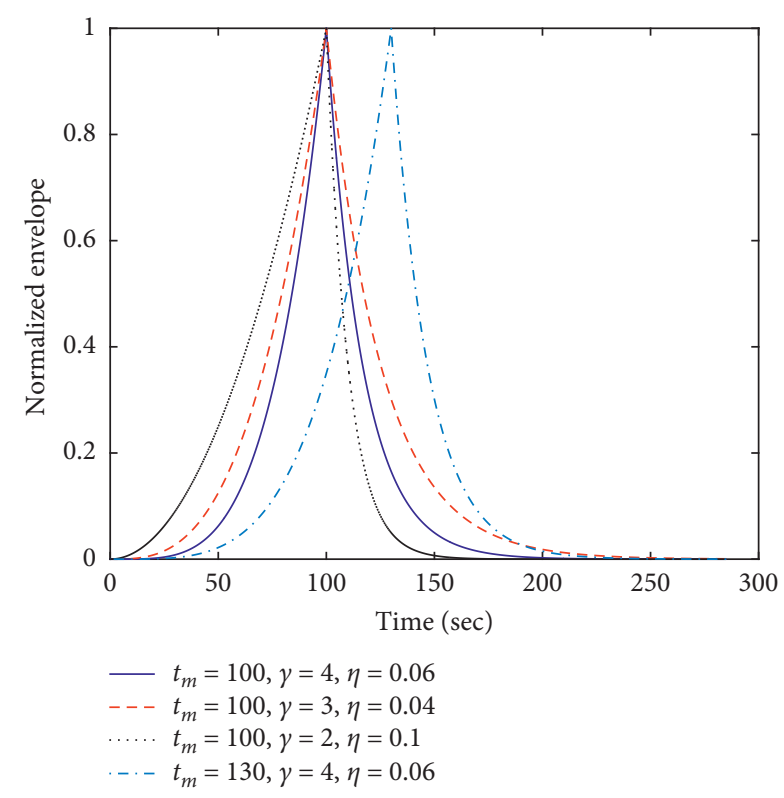

FIGURE 10: Piecewise function with various parameters of $t_{m}, \gamma$, and $\eta$.

TABle 3: Parameters controlling envelope of motions.

\begin{tabular}{lccc}
\hline Record & $t_{0}$ & $\gamma$ & $\eta$ \\
\hline CHB002EW & 0.011 & 4.45 & 0.061 \\
TKY023EW & 22.61 & 1.96 & 0.030 \\
SIT003EW & 0.048 & 2.68 & 0.019 \\
\hline
\end{tabular}

4.1.2. Modification in Frequency Domain. Wavelet coefficients in time-frequency domain comply with a bivariate lognormal distribution, which is used to present the variability of ground motions [10]. To simplify this, a set of shaped-like lognormal functions are used to describe the power spectrum density for each column (time instant). Thus, a set of squared wavelet coefficients derived from the modulated Gaussian process are modified by multiplying each fitted function to simulate the variability of targeted motions.

The shaped-like lognormal function of the $k^{\text {th }}$ column with respect to frequency $f$ is given as follows:

$$
L_{k}\left(f \mid u_{k}, \sigma_{k}\right)=\frac{1}{f \sigma_{k} \sqrt{2 \pi}} \exp \left(\frac{-\left(\ln f-u_{k}\right)^{2}}{2 \sigma_{k}^{2}}\right),
$$

where the parameters of $\mu_{k}$ and $\sigma_{k}$ for the $k^{\text {th }}$ column are attained by fitting the assigned function to squared coefficients at the $k^{\text {th }}$ column in the sense of power energy and $L_{k}$ represents the power spectrum at the $k^{\text {th }}$ column.

As a result, normalized squared coefficients for specific columns of CHB002EW and TKY023EW are shown in Figure 12, together with the fitted functions. The fitted models generally reflect the distribution of the power spectra because their energy tends to concentrate at low frequencies as time columns evolve.

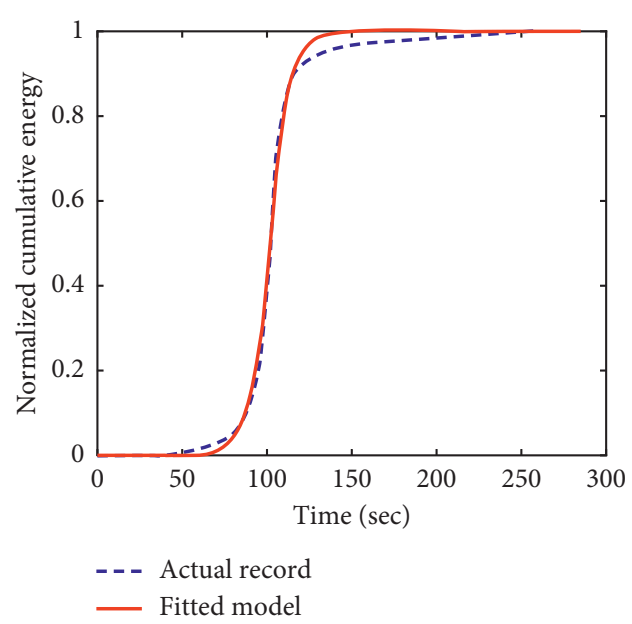

(a)

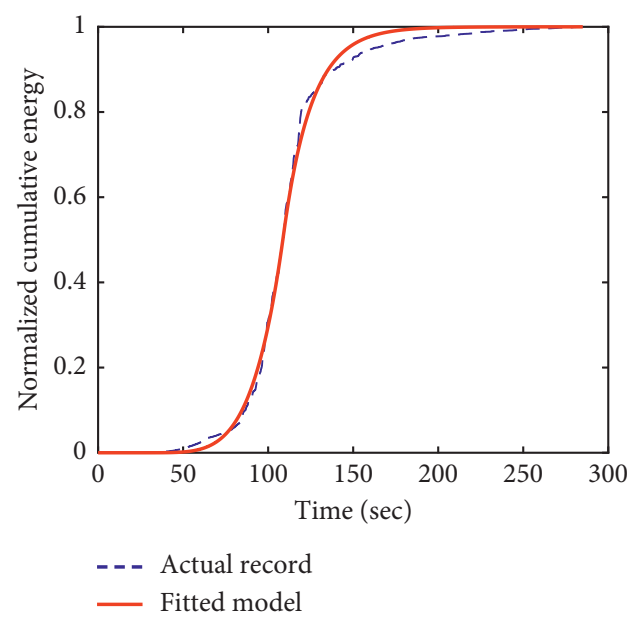

(b)

FIGURE 11: Normalized cumulative energy curves for records and modulated Gaussian process. (a) CHB002EW and (b) TKY023EW.

To establish a parametric relationship between the identified parameters and the sequence of columns (time instants), a generic expression built on the superposition of Gaussian functions is defined as follows:

$$
\lambda_{k}(k)=\sum_{i} s_{i} \exp \left[-\left(\frac{\left(k-\varepsilon_{i}\right)}{\psi_{i}}\right)^{2}\right],
$$

where subscript $i$ indicates the $i^{\text {th }}$ Gaussian function; $s_{i}$ is the scaling factor; $\varepsilon_{i}$ indicates the location for peak value; $\psi_{i}$ denotes the width of "bell" Gaussian function; and $\lambda_{k}$ represents the variables of $\mu_{k}$ and $\sigma_{k}$ in the $k^{\text {th }}$ column, respectively. The superposition of two and three Gaussian functions are used to model the variables of $\mu_{k}$ and $\sigma_{k}$, respectively. The fitted results are listed in Tables 4 and 5 .

As illustrated in Figure 13, it is found that the parameter $\mu$ decreases with an increase in the sequence of columns in general. This is consistent with the fact that high-frequency components mainly appear at beginning, thus accounting for great contributions to the intensity of a ground motion; at the same time, low-frequency components gradually 

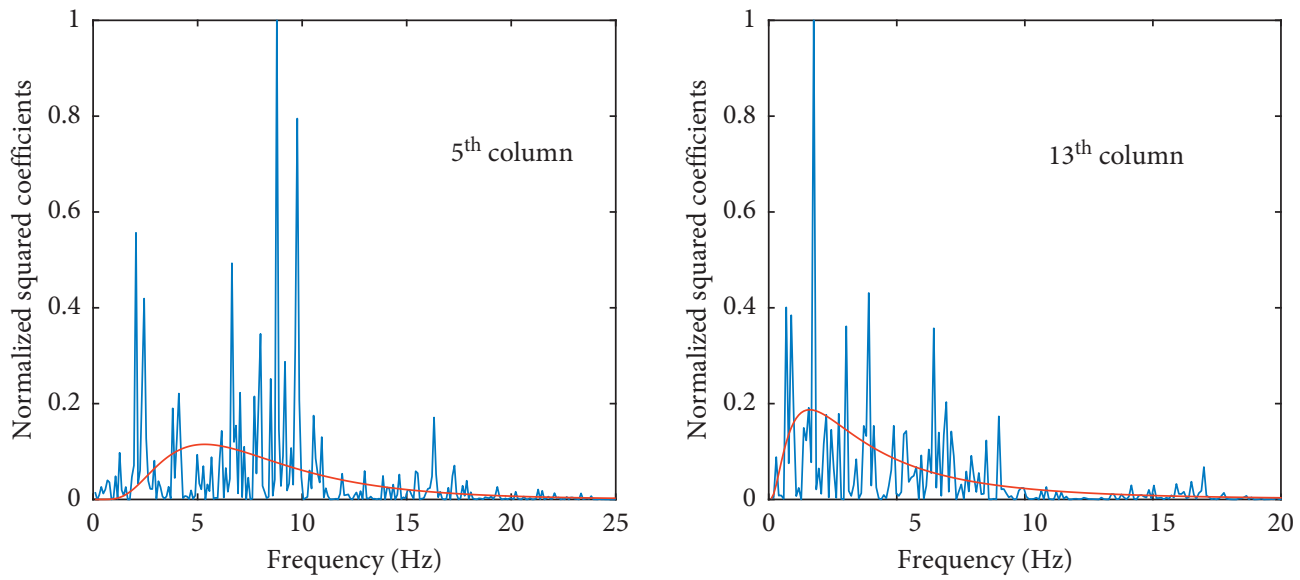

- Actual record

— Fitted model

- Actual record

— Fitted model
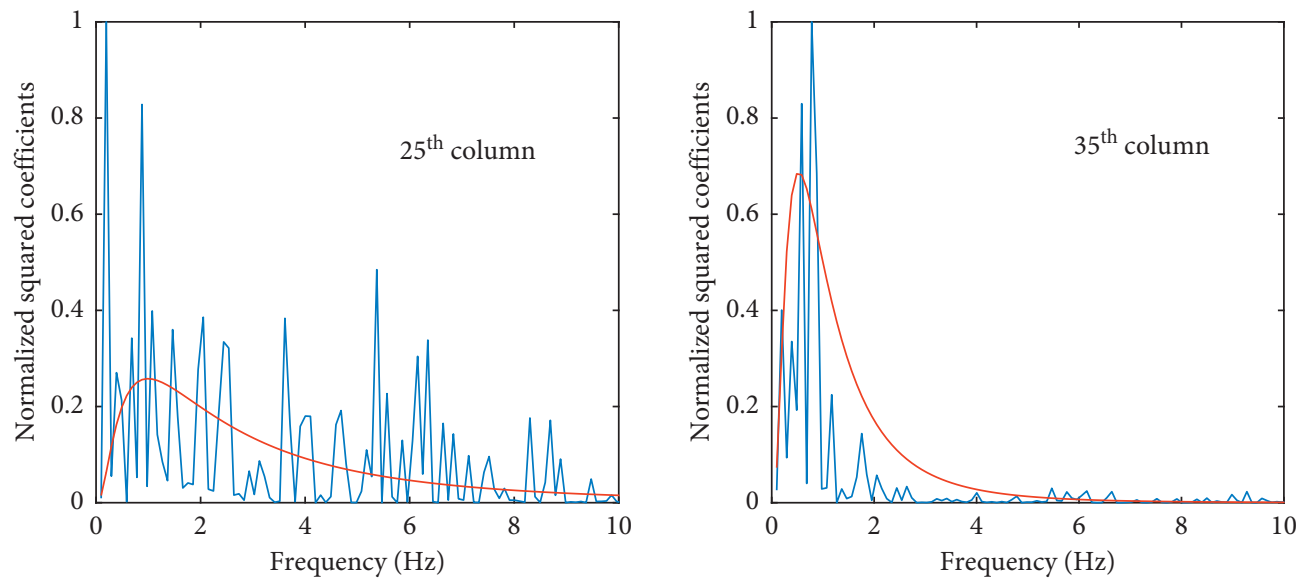

- Actual record

Fitted model

- Actual record

— Fitted model

(a)

Figure 12: Continued. 

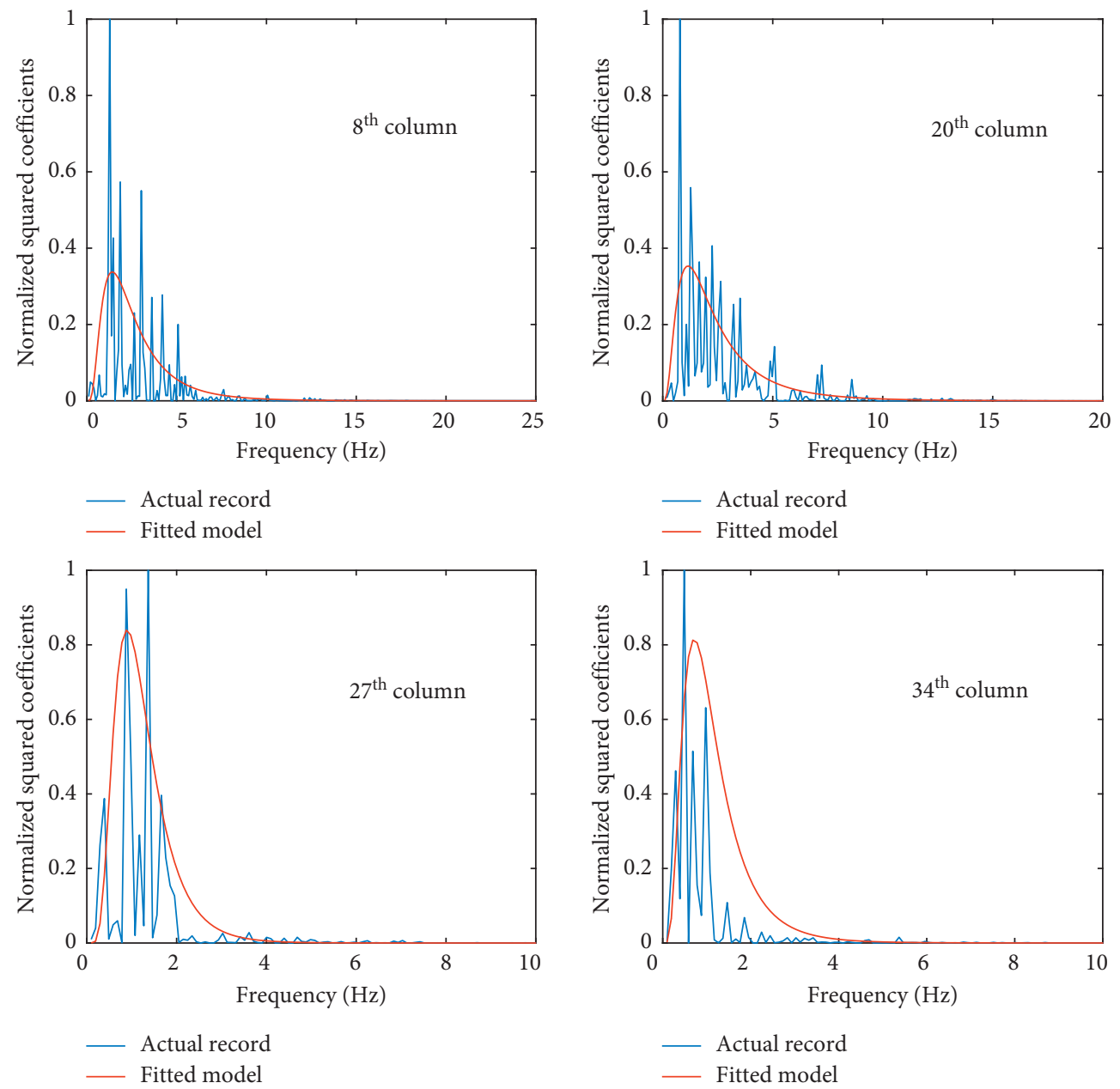

(b)

FIGURE 12: Comparison of the fitted models with actual records in terms of normalized power spectrum at specific columns. (a) CHB002EW and (b) TKY023EW.

TABLE 4: Parameters controlling $\mu$ for each column.

\begin{tabular}{lcccccc}
\hline Record & $s_{1}$ & $\varepsilon_{1}$ & $\psi_{1}$ & $s_{2}$ & $\varepsilon_{2}$ \\
\hline CHB002EW & 2.28 & 1.45 & 11.88 & 1.52 & 19.99 & 6.88 \\
TKY023EW & 1.52 & -13.04 & 23.90 & 0.47 & 19.26 & 2.95 \\
SIT003EW & 31.65 & -46.63 & 27.61 & 0.80 & 19.56 \\
\hline
\end{tabular}

TABLE 5: Parameters controlling variance $\sigma$ for each column.

\begin{tabular}{|c|c|c|c|c|c|c|c|c|c|}
\hline Record & $s_{1}$ & $\varepsilon_{1}$ & $\psi_{1}$ & $s_{2}$ & $\varepsilon_{2}$ & $\psi_{2}$ & $s_{3}$ & $\varepsilon_{3}$ & $\psi_{3}$ \\
\hline CHB002EW & 1554.10 & 145.98 & 33.68 & -0.73 & 21.16 & 4.38 & 1.24 & 24.87 & 21.47 \\
\hline TKY023EW & 0.69 & 0.47 & 10.11 & 0.39 & 16.33 & 5.97 & 1.00 & 89.04 & 65.94 \\
\hline SIT003EW & 1555.50 & 154.65 & 21.78 & -8.26 & 7.78 & 41.05 & 9.07 & 5.26 & 45.48 \\
\hline
\end{tabular}

dominate over high-frequency components as time evolves. In contrast, the trend for the parameter $\sigma$ appears to be complicated. It is possible that variations in bandwidth among time instants result in the variability of the time-frequency domain. This trend is similar to that depicted by using the lognormal probability distribution in paper [30].

It is shown from Figure 14 that the stationary property of the Gaussian random process is transformed into nonstationary property of the initial seed motions. 

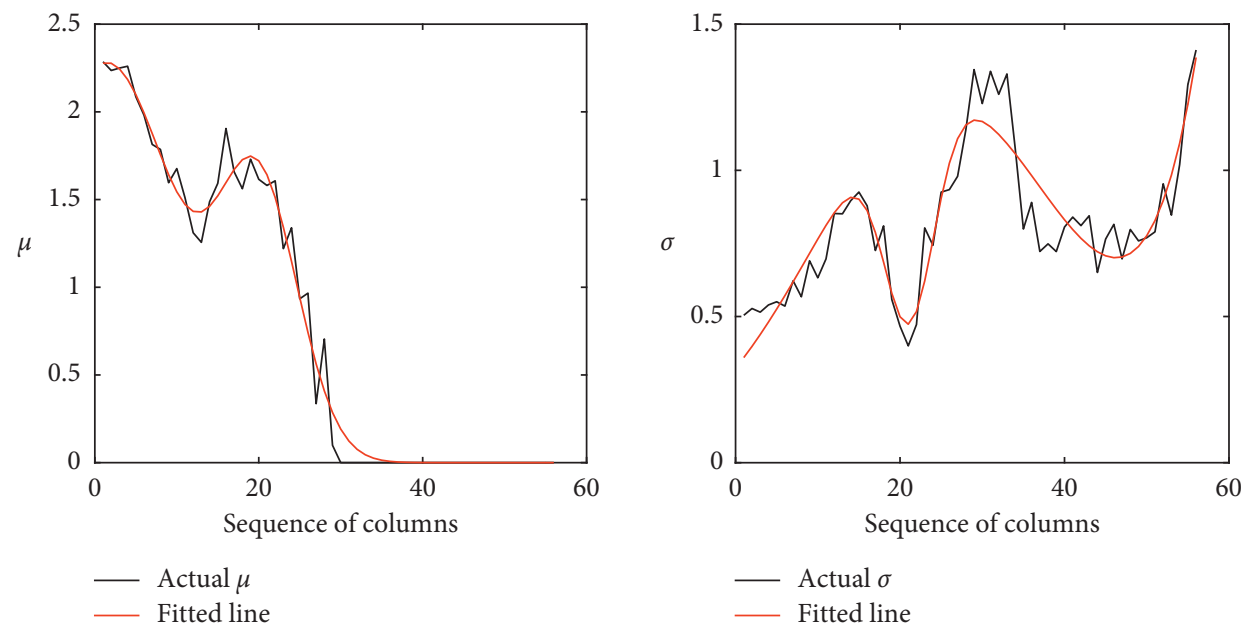

(a)
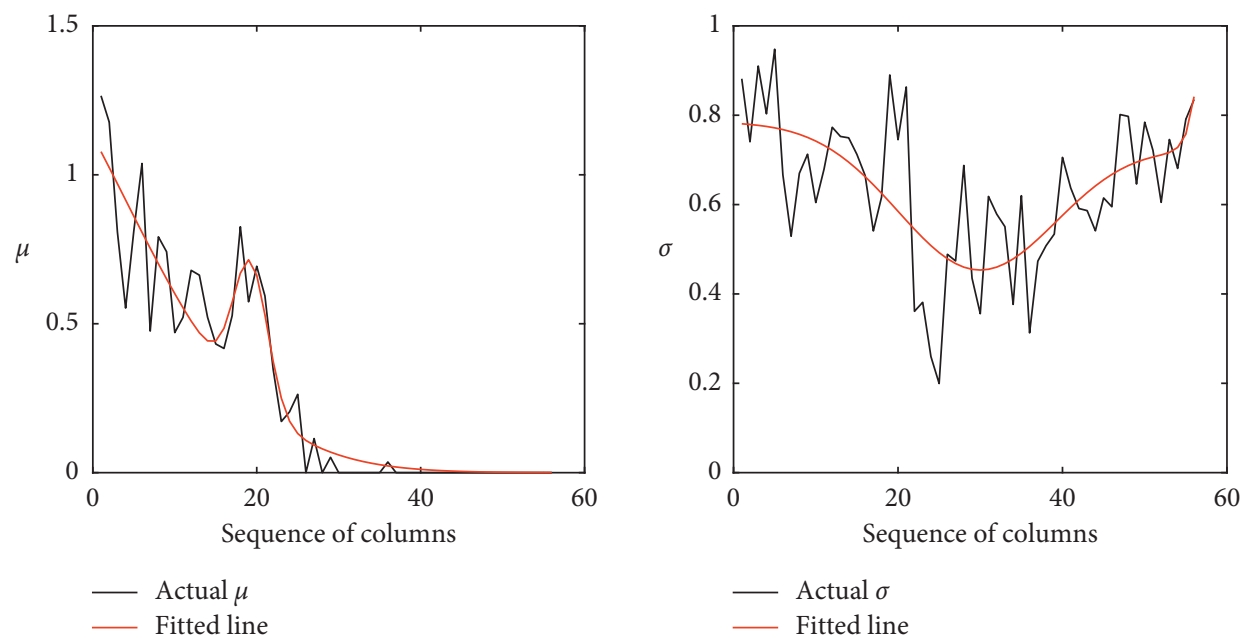

(b)

FIGURE 13: Variations of parameters $\mu_{k}$ and $\sigma_{k}$ with respect to time instant. (a) CHB002EW and (b) TKY023EW.

Furthermore, the properties of the initial seed motions are similar to those of their target motions, which are illustrated in Figure 15; note that this result is detailed later.

4.2. Simulation of High-Frequency Components Compatible with Target Motions. The methodology related to compatibility is that wavelet coefficients are scaled up or down among rows on the basis of the linear relationship between amplitudes of frequency components and an elastic response of a single degree of freedom system; meanwhile, coefficients are scaled among columns based on the squared relationship between amplitudes and cumulative energy [11].

4.2.1. Compatibility with Frequency Response. Specifically, the scale factor ${ }^{n+1} c_{j, k}^{i}$ is determined from the ratio of the targeted acceleration spectrum $\operatorname{Sa}_{\text {target }}\left(f_{i}\right)$ to the simulated spectrum $\operatorname{Sa}_{\text {simulated }}^{n}\left(f_{i}\right)$ at frequency $f_{i}=i d f_{w}$. Then, the new time series ${ }^{n+1} \boldsymbol{s}(t)$ is readily available from the inverse wavelet transform after the $(n+1)^{\text {th }}$ iteration. The iteration process is performed until the mean error is satisfied with the assigned threshold. Accordingly, the equations related to adjustment in frequency domain are expressed as follows:

$$
\begin{aligned}
{ }^{n+1} c_{j}^{i} & ={ }^{n} c_{j, k}^{i} \frac{\operatorname{Sa}_{\text {target }}\left(f_{i}\right)}{\operatorname{Sa}_{\text {simulated }}^{n}\left(f_{i}\right)}, \quad i=1,2, \ldots, 2^{j}, \\
{ }^{n+1} s(t) & =\sum_{i=1}^{2^{j}} \sum_{k=1}^{2^{N-j}}{ }^{n+1} c_{j, k}^{i} \psi_{j, k}^{i}(t) .
\end{aligned}
$$

It is essential to locate the instant time $t_{k}$, at which the $5 \%$ damping single degree of the freedom oscillator with natural frequency $f_{i}$ presents the maximum response. This enables us to merely modify the amplitudes with centre frequency $f_{i}$ at the $k^{\text {th }}$ column instead of modifying the amplitudes with centre frequency $f_{i}$ over entire columns. In addition, the instant time $t_{k}$ should be within $\left[0, t_{l}\right]$. This is in accordance with the result of phase dispersion that the high-frequency components arrive earlier than long-period components. 

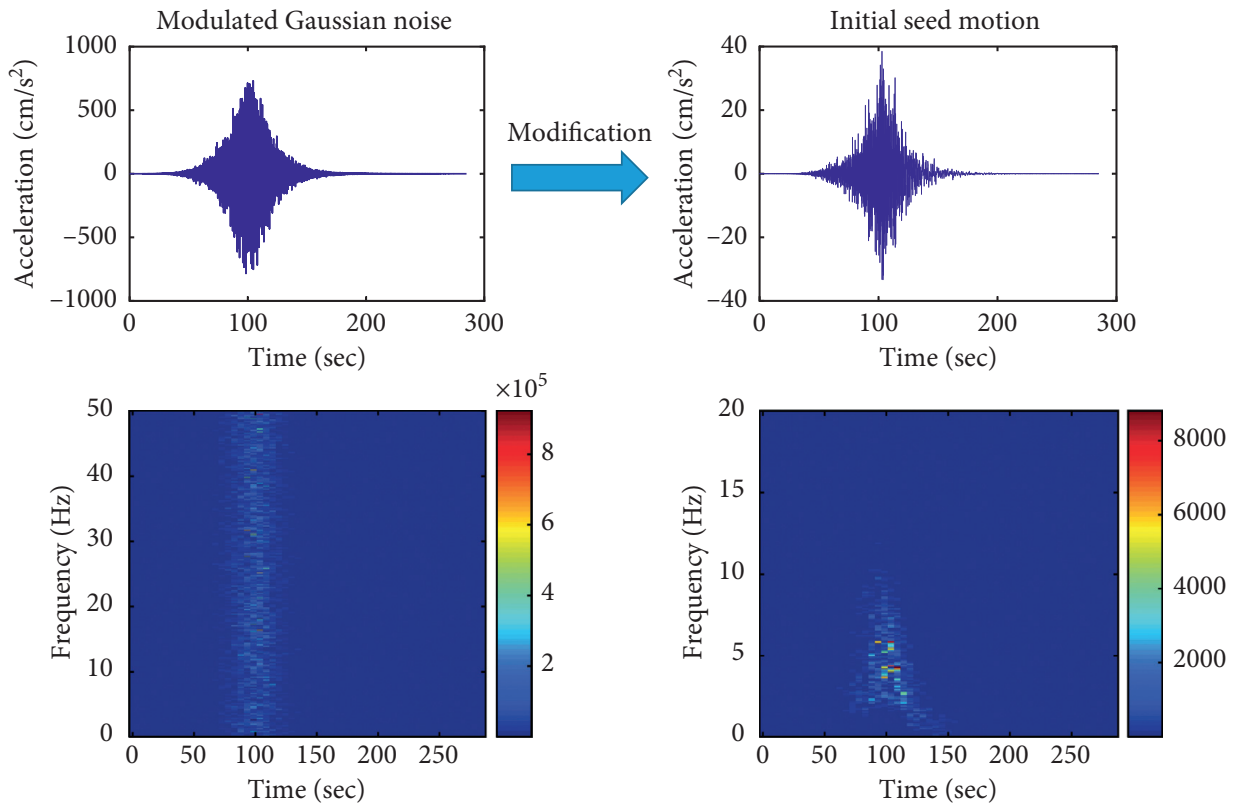

(a)
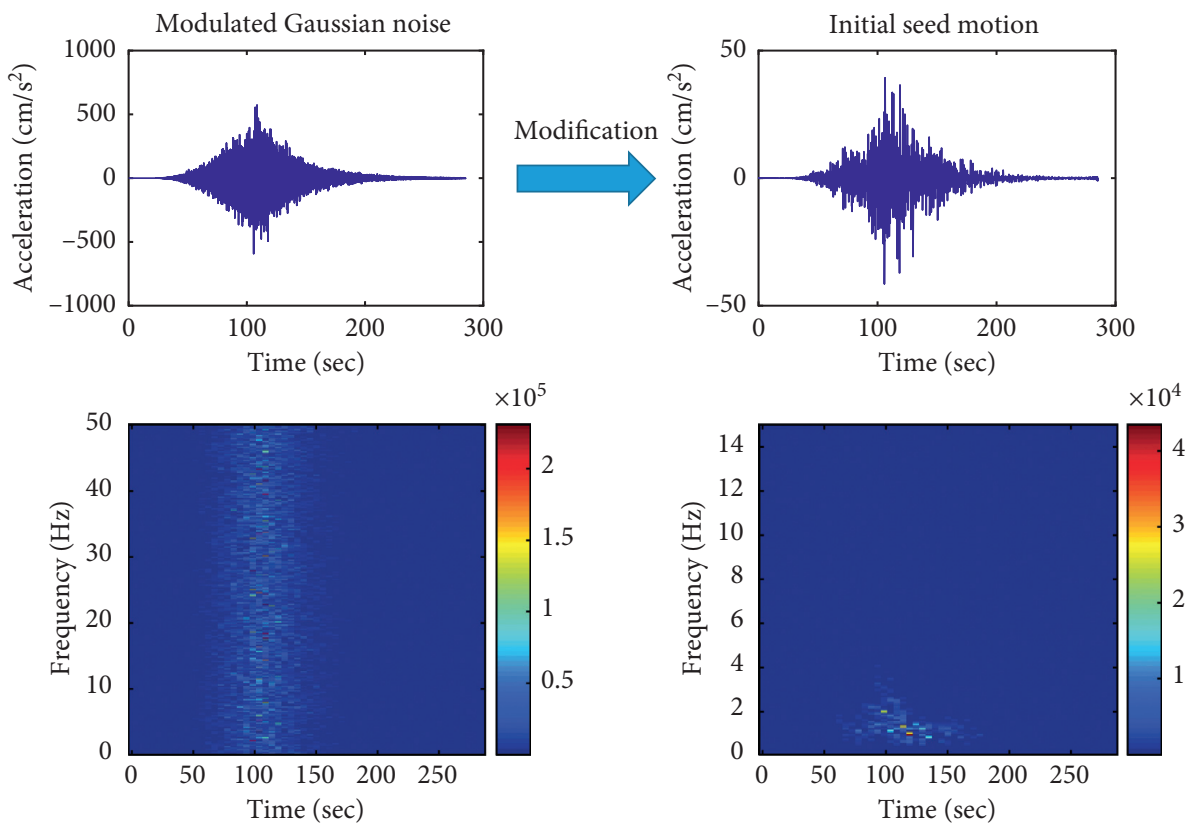

(b)

FIGURE 14: Illustration of changes in the initial seed motions considering the time-frequency domain. (a) CHB002EW and (b) TKY023EW.

As suggested by the limitation of wavelet packets, there is low resolution at long periods. This means that the procedure cannot attain adequate accuracy during long periods ranging from 5 to $10 \mathrm{sec}$. Because of this, the frequency range in this process is defined within $[0.3,25 \mathrm{~Hz}]$, during which wavelet packets take advantage of high resolution to make simulations compatible with the targeted spectrum as accurately as possible.

4.2.2. Compatibility with Cumulative Energy. The ratio ${ }^{n+1} w(t)$ for modification in time domain is calculated from the squared root of the incremental energy curve at the time interval $\left[t_{k-1}, t_{k}\right]$, and its expression is given as follows:

$$
\begin{array}{r}
n+1 \\
w\left(t_{k}\right)=\sqrt{\frac{H_{\text {target }}\left(t_{k}\right)-H_{\text {target }}\left(t_{k-1}\right)}{{ }^{n} H_{\text {simulated }}\left(t_{k}\right)-{ }^{n} H_{\text {simulated }}\left(t_{k-1}\right)}}, \\
k=1,2, \ldots, \frac{N}{2^{j}} .
\end{array}
$$

As mentioned in equation (13), a small frequency interval $\mathrm{d} f_{w}=\mathrm{d} t / 2^{j+1}$ gives rise to a large time interval $\mathrm{d} t_{w}=2^{j} \mathrm{~d} t$. Because of this, the ratios ${ }^{n+1} w(t)$ are 

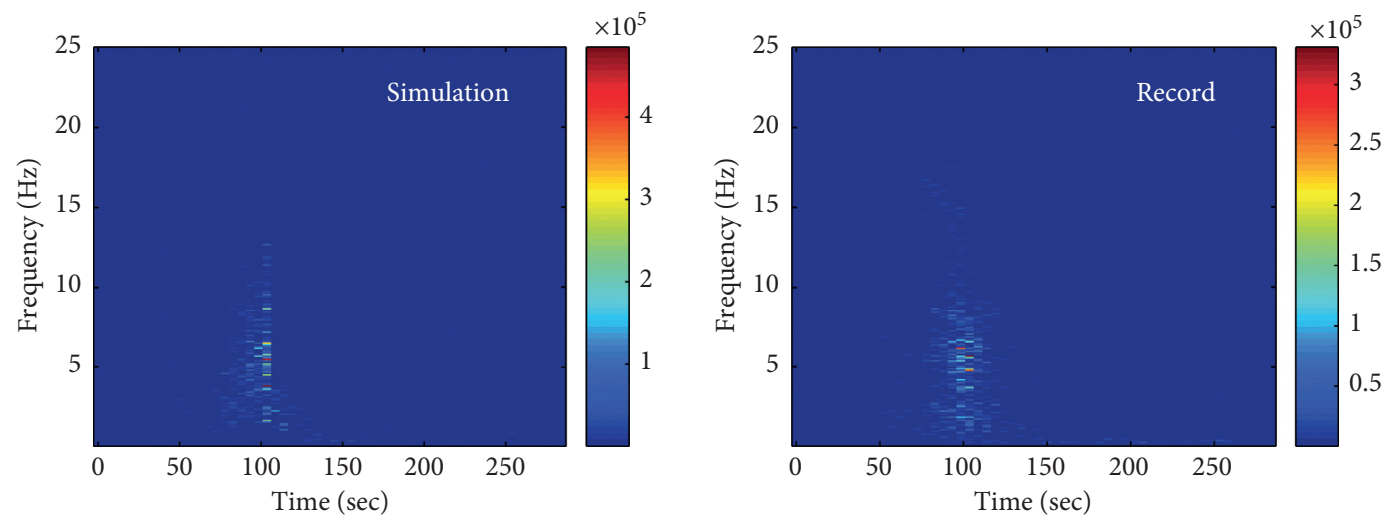

(a)
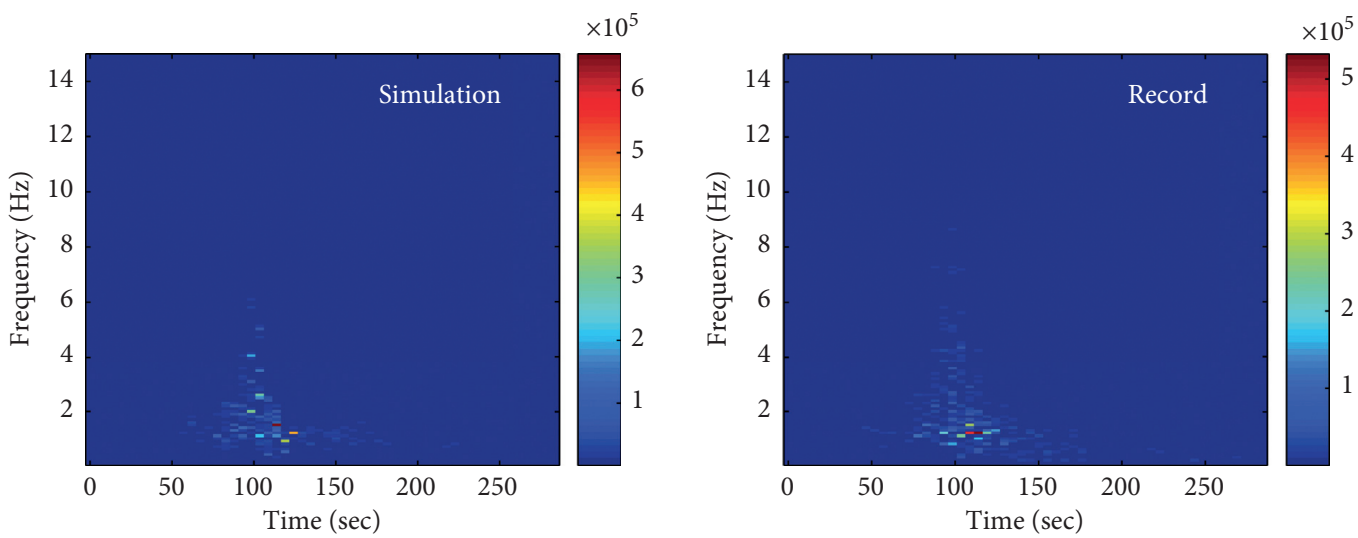

(b)

FIGURE 15: Comparison of the simulations with the records with respect to wavelet coefficients. (a) CHB002EW and (b) TKY023EW.

interpolated by using a cubic spline to obtain the ratios ${ }^{n+1} w(t)^{\prime}$ at each time interval $\mathrm{d} t$. Then, a new time series ${ }^{n+2} s(t)$ is generated after multiplying the ratios ${ }^{n+1} w(t)^{\prime}$. Its expression is written in equation (23). Note that the new series ${ }^{n+2} s(t)$ should be baseline corrected through a highpass filter with a low cutoff frequency of 0.1 :

$$
{ }^{n+2} s(t)={ }^{n+1} w(t)^{\prime n+1} s(t) .
$$

Consequently, it is found from Figure 16 that the simulated acceleration spectra present close agreement with targeted motions, except for the longer period range (5-10 sec); for cumulative energy curves, the simulations nearly overlap with the records, suggesting that they share the same acceleration waveform. In addition, as illustrated in Figure 15, the simulations appear to be the similar distribution of wavelet coefficients to the records, since the majority of the large coefficients of the simulations are consistent with that of the records.

As suggested by Huang [11], two mean lognormal errors are employed to evaluate the accuracy of compatibility with the acceleration spectrum and cumulative energy curve, respectively. After 20 iterations in Figure 17, the errors for spectrum and cumulative energy curve are almost below 0.02 and 0.001 , respectively.

Accordingly, two samples of simulated high-frequency components are displayed in the form of the acceleration and velocity time series in Figure 18. In addition, the velocity spectra attained from their acceleration time series are presented in Figure 19. The velocity spectra of simulated motions are similar to their original records at low to intermediate periods; however, the spectra of the original records at longer periods are significantly larger than those of the simulated motions. This means that the simulated motions are characterized by high-frequency components relative to their original records.

\section{Combination and Verification}

After the long-period and high-frequency components are simulated individually, they are incorporated into each other in time domain. Accordingly, Figure 20 shows four samples of the simulations for the records CHB002EW and TKY023EW. From visual inspection, four realizations are similar to their actual records in terms of acceleration waveforms, since their strong shaking process occurs at approximately $100 \mathrm{sec}$, which is consistent with the actual records seen in Figure 2. In addition, the obvious presence of LALP surface waves in simulated velocity waveforms highlights their long-period properties. However, it is noted that the components with an estimated period of $20 \mathrm{sec}$ are absent from the simulated velocity series. This results from the low resolution of wavelet packets in discerning 

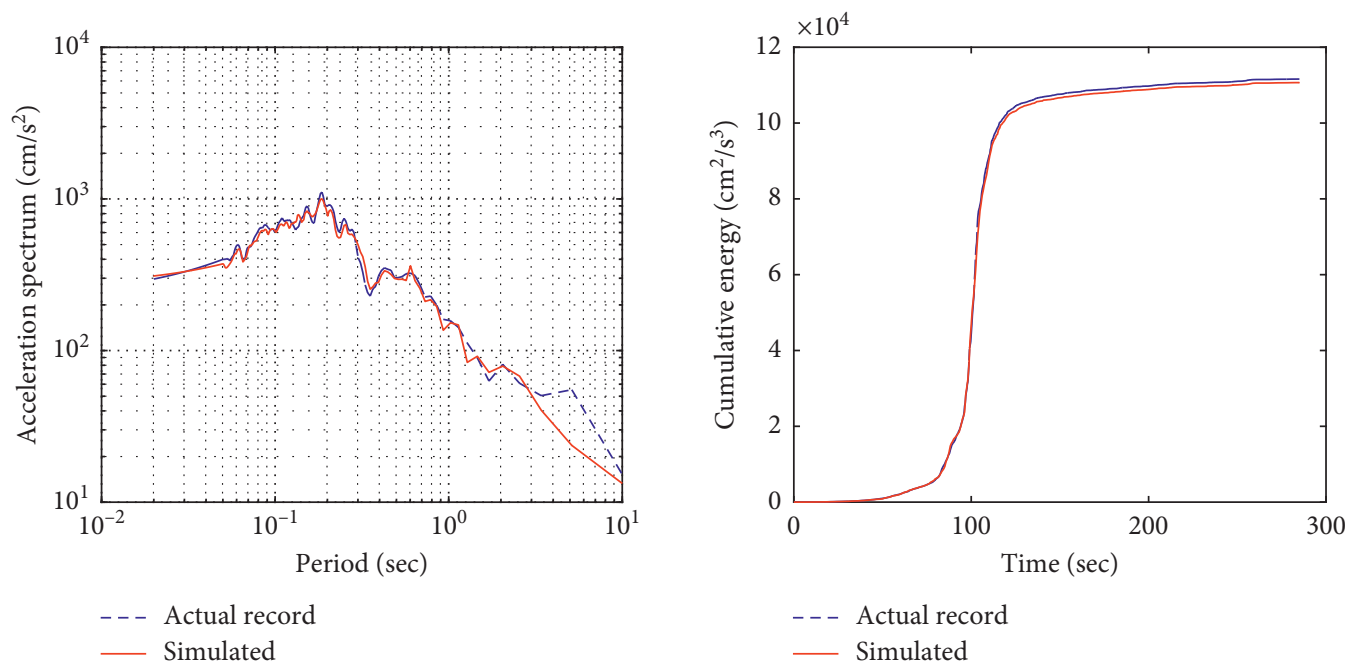

(a)
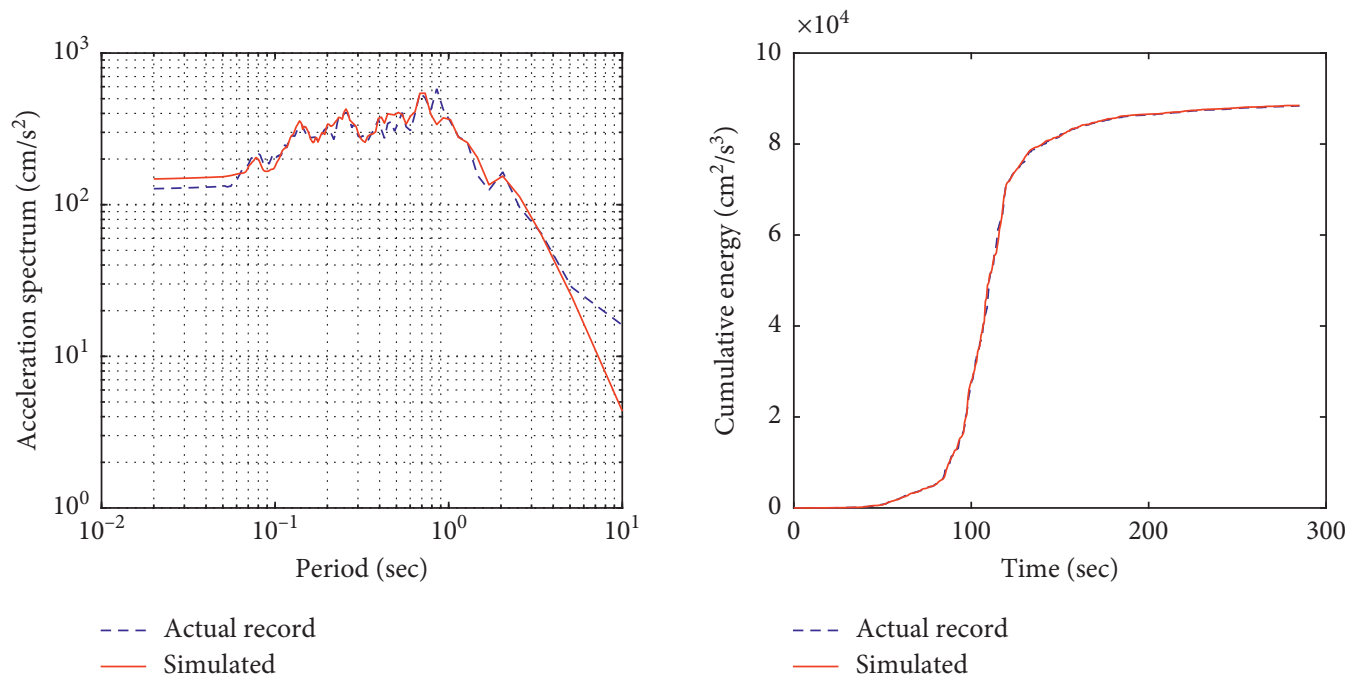

(b)

Figure 16: Comparison of simulated high-frequency components with the records with respect to response spectrum and cumulative energy curve. (a) CHB002EW and (b) TKY023EW.

components with periods larger than $10 \mathrm{sec}$. However, it seems that such long-period components are not of interest in engineering practice because the resonant response of structures is mainly attributed to the components with periods ranging from 5 to $10 \mathrm{sec}$ [31].

Then, comparisons of four realizations with actual records in terms of the cumulative energy curve and Fourier amplitude spectrum are illustrated in Figure 21. It is clear that each sample matches well with the actual records considering the cumulative energy curve. In addition, the Fourier amplitude spectra of the realizations are close to their actual records over frequencies ranging from $0.1 \mathrm{~Hz}$ to $20 \mathrm{~Hz}$. However, distinct discrepancies appear at frequencies below $0.1 \mathrm{~Hz}$, resulting from the limitations of wavelet packets.

Furthermore, variations of the simulated long-period motions are mainly dependent on the simulated long-period components generated by means of spectral representation, when the simulated long-period motions are verified in sense of compatibility with a target response spectrum.

In this regard, 100 realizations of long-period components are individually incorporated with one simulated high-frequency motion which consists of high-frequency components in order to form a set of simulated long-period motions, for which 100 response spectra with 5\% damping are attained. As shown in Figure 22, the median spectra of the simulated motions are closely aligned with the target spectra. Two dominant periods for the high-frequency and long-period components, each of which are 0.2 and $5.3 \mathrm{sec}$ for CHB002EW and 0.7 and $6.6 \mathrm{sec}$ for TKY023EW, are captured well by the median simulations. This suggests that the simulated long-period motions replicate the properties of the actual motions. Meanwhile, it is observed that the actual spectra at loner periods from 4 to $10 \mathrm{sec}$ mostly fall 


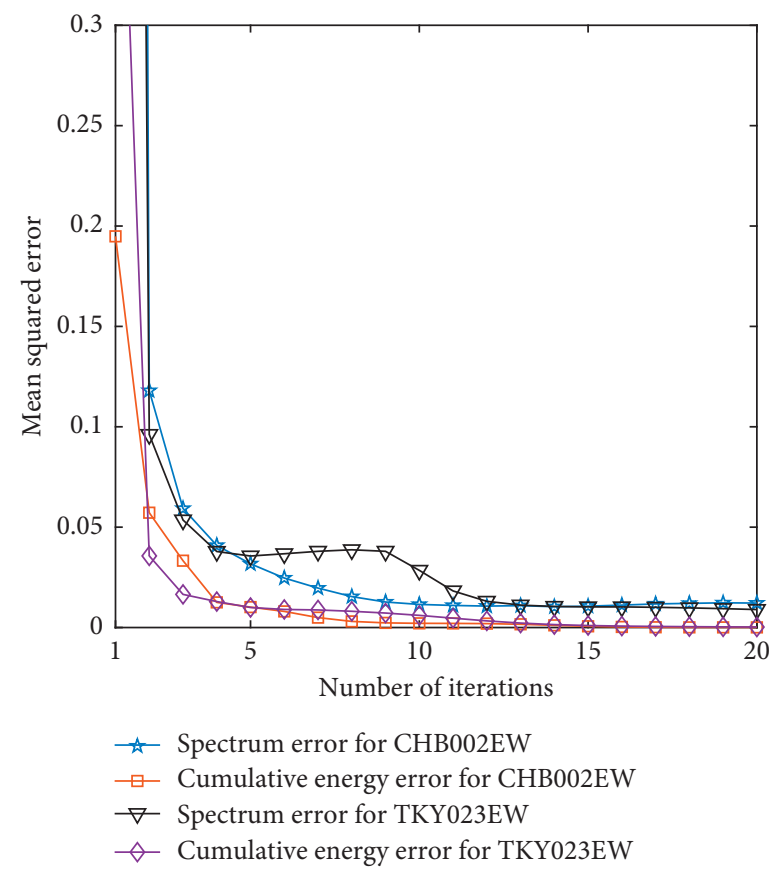

FIGURE 17: Mean squared error for each iteration.
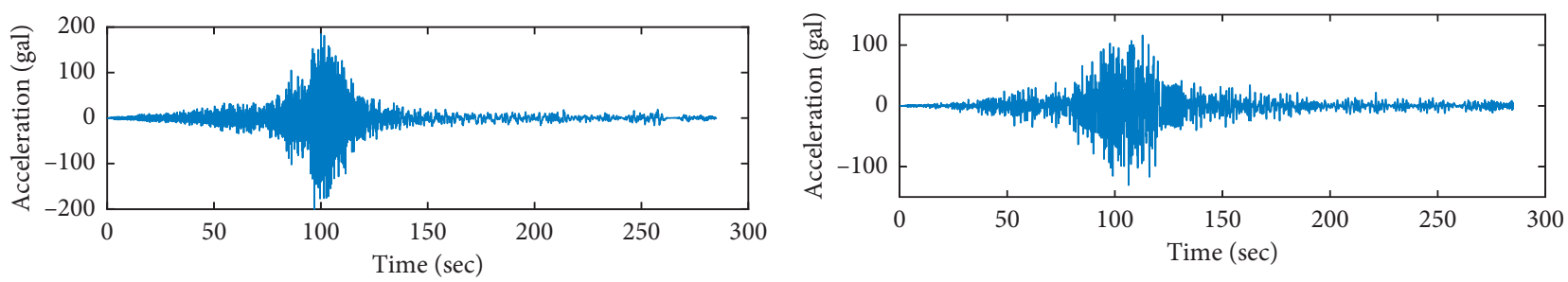

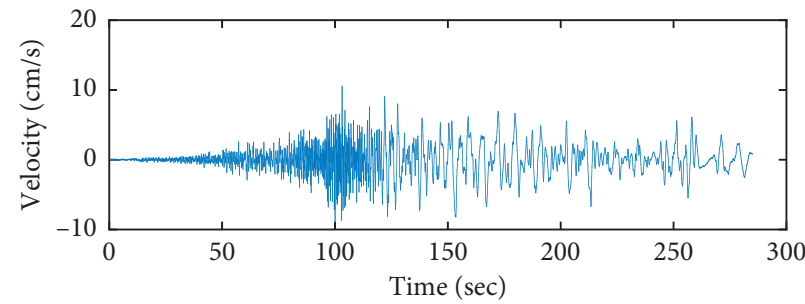

(a)

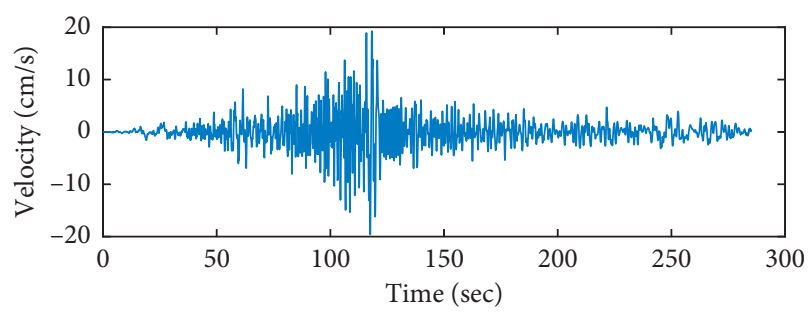

(b)

FIGURE 18: Simulations of acceleration and velocity time series by means of wavelet packets. (a) CHB002EW and (b) TKY023EW.

within the bound of the standard deviation $\pm \sigma$, and thus, this means that the actual motions could be one of the ensembles of the simulated motions in the stochastic sense.

\section{Summary}

In this paper, a method for generating the long-period ground motions based on the combination of spectral representation and wavelet packets is proposed. A flowchart regarding this method is illustrated in Figure 23. It is clear that the proposed method is divided into three parts: (I) spectral representation for long-period components, (II) simulation of high-frequency components using wavelet packets, and (III) superposition of the former into the latter in time domain. To depict clearly these procedures, an illustrative example for SIT003EW, which is another record in Tohuku earthquake M9, is presented in Figure 24 as follows.

For the first phase, the LALP velocity surface waves are truncated from the real motion with reference to $t_{l}$ shown in Figure 24(a), conditioned by the truncated segment showing an agreement with its original record displayed in Figure 24(b); the power spectrum density for the truncated velocity segment is estimated shown in Figure 24(c); instantaneous amplitudes of the velocity 


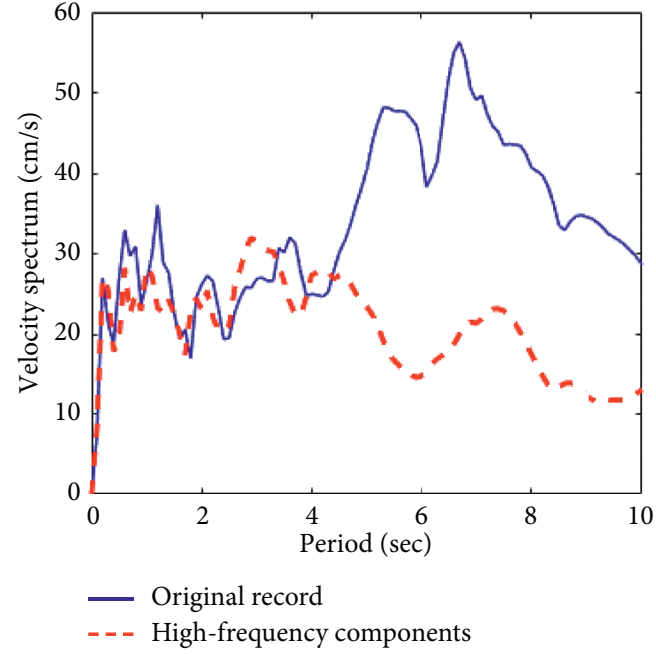

(a)

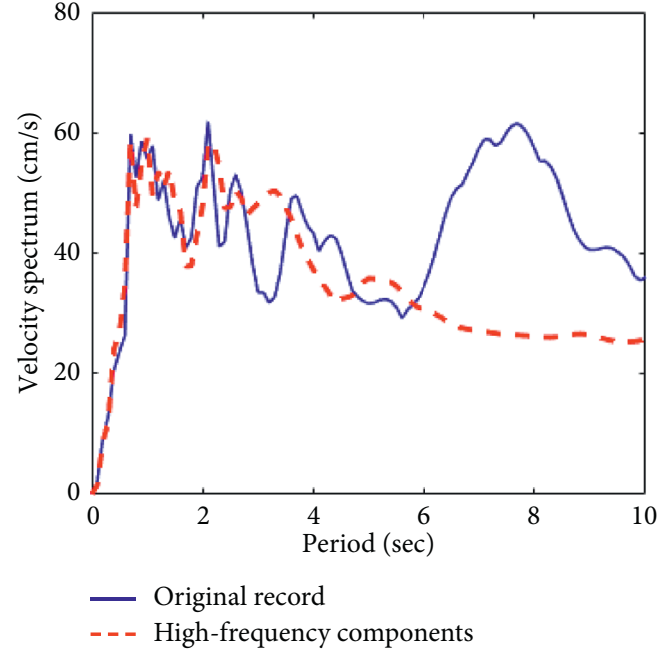

(b)

FIgURE 19: Comparison of original records with the simulations in terms of velocity spectrum. (a) CHB002EW and (b) TKY023EW.
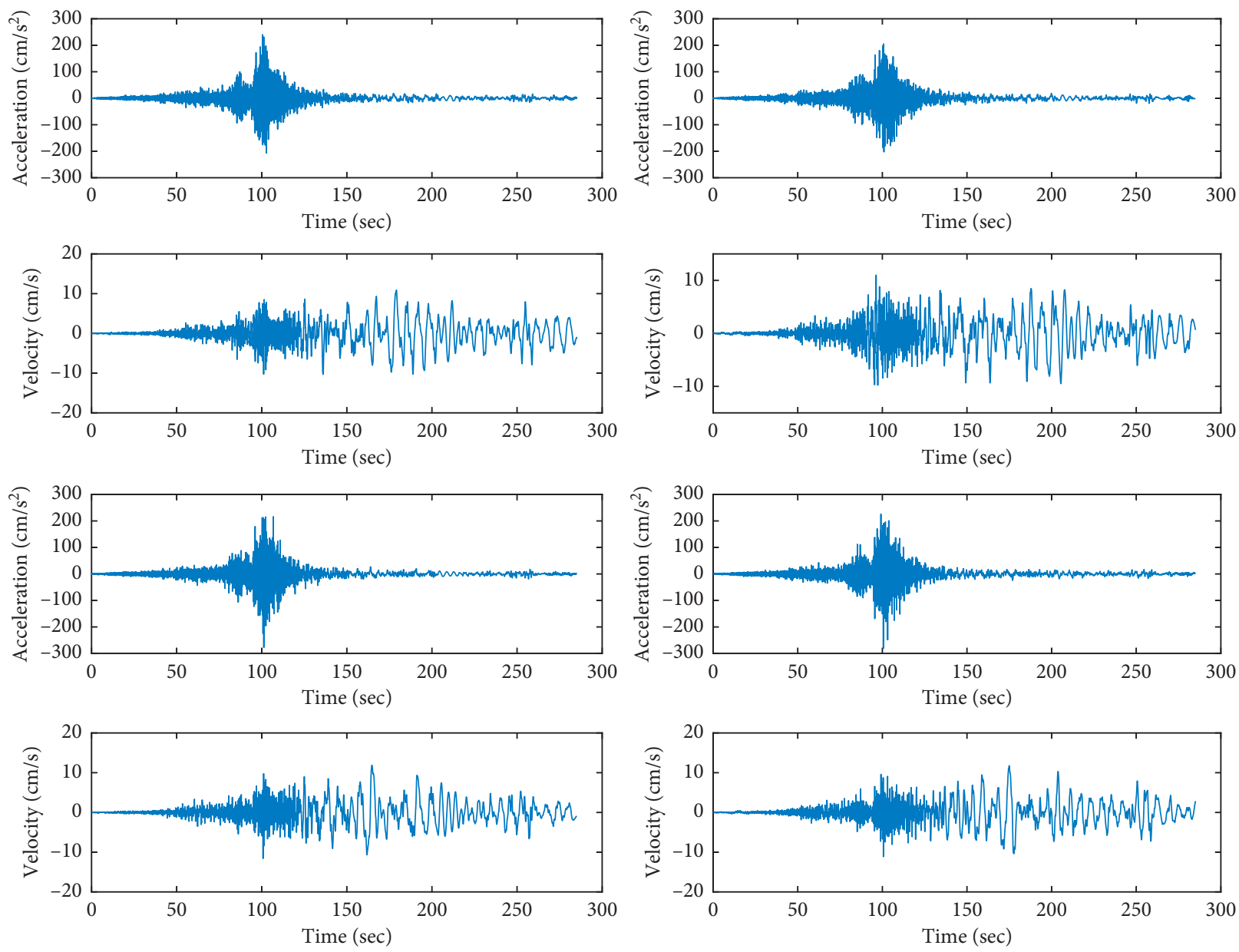

(a)

Figure 20: Continued. 

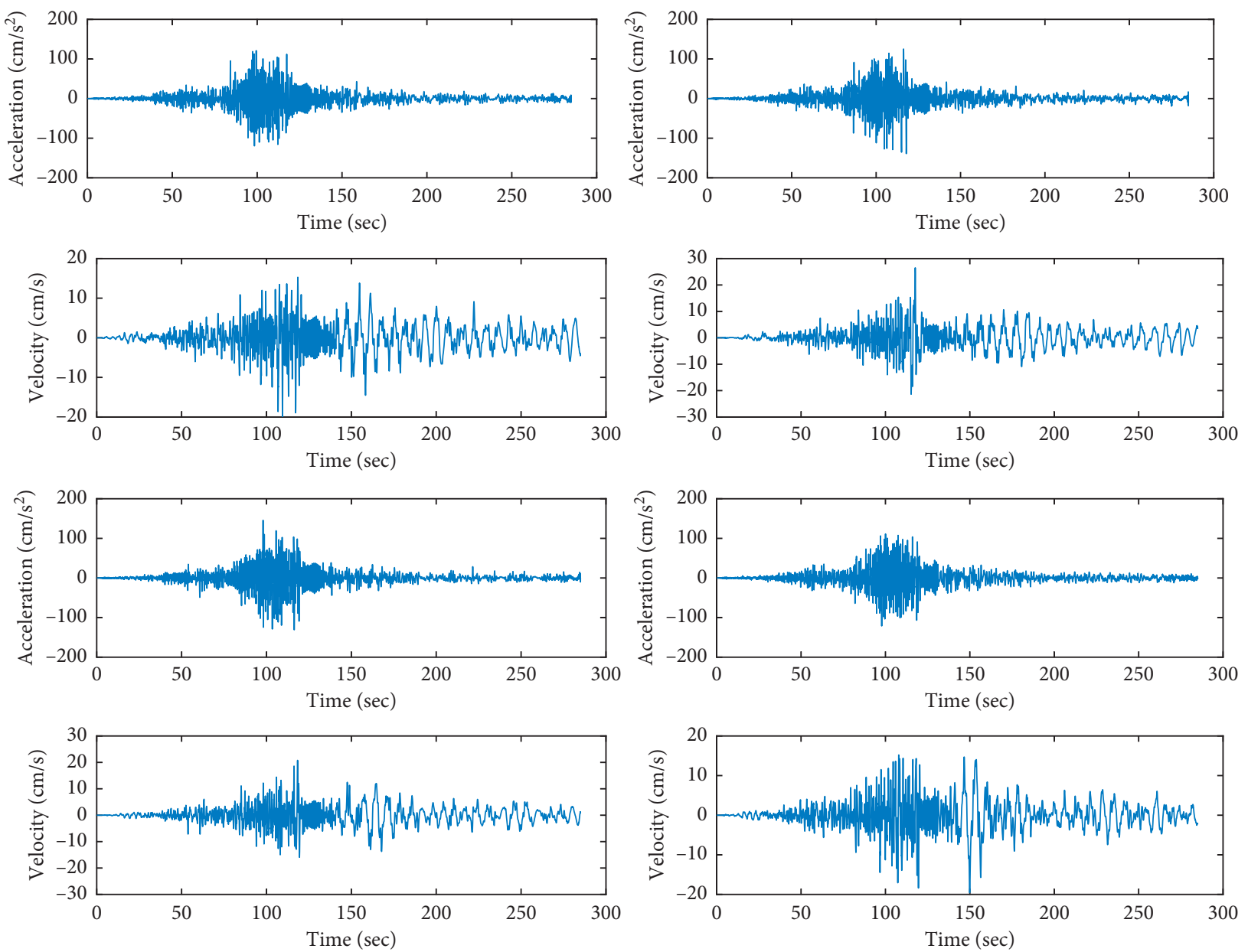

(b)

Figure 20: Samples of the simulations in the form of acceleration and velocity time series. (a) CHB002EW and (b) TKY023EW.
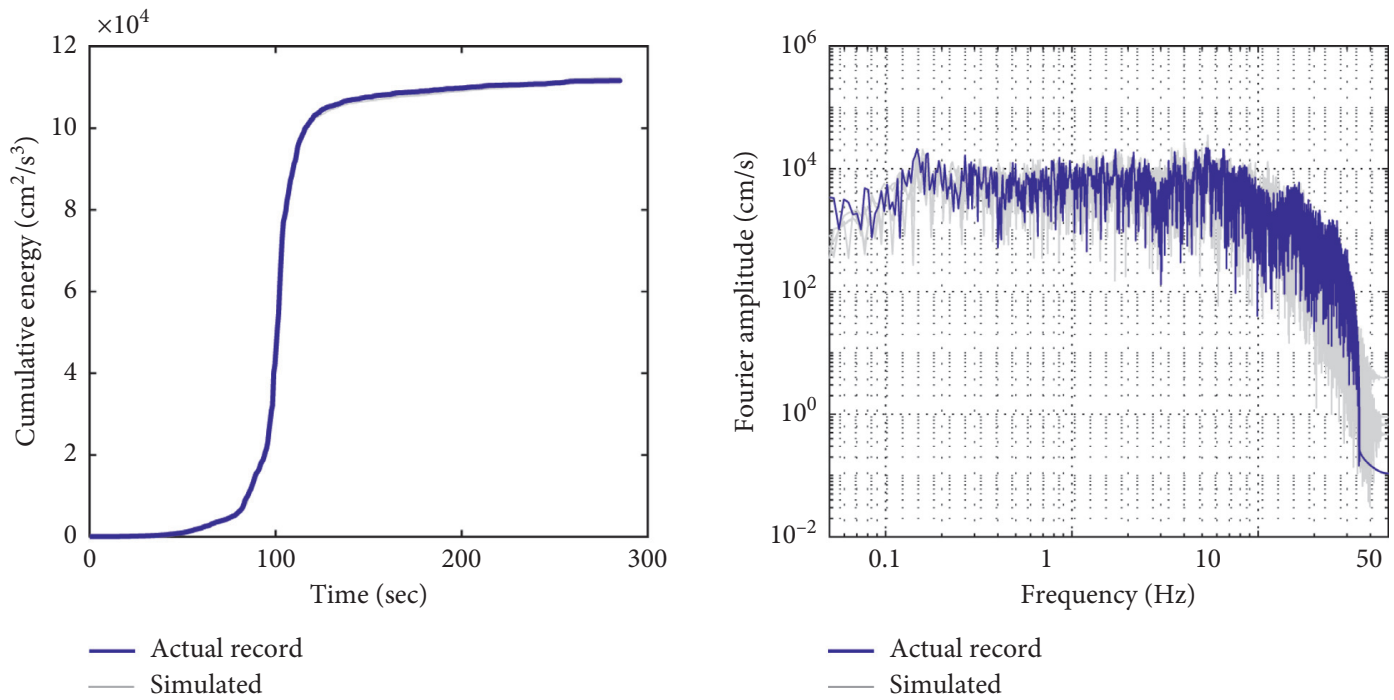

(a)

Figure 21: Continued. 

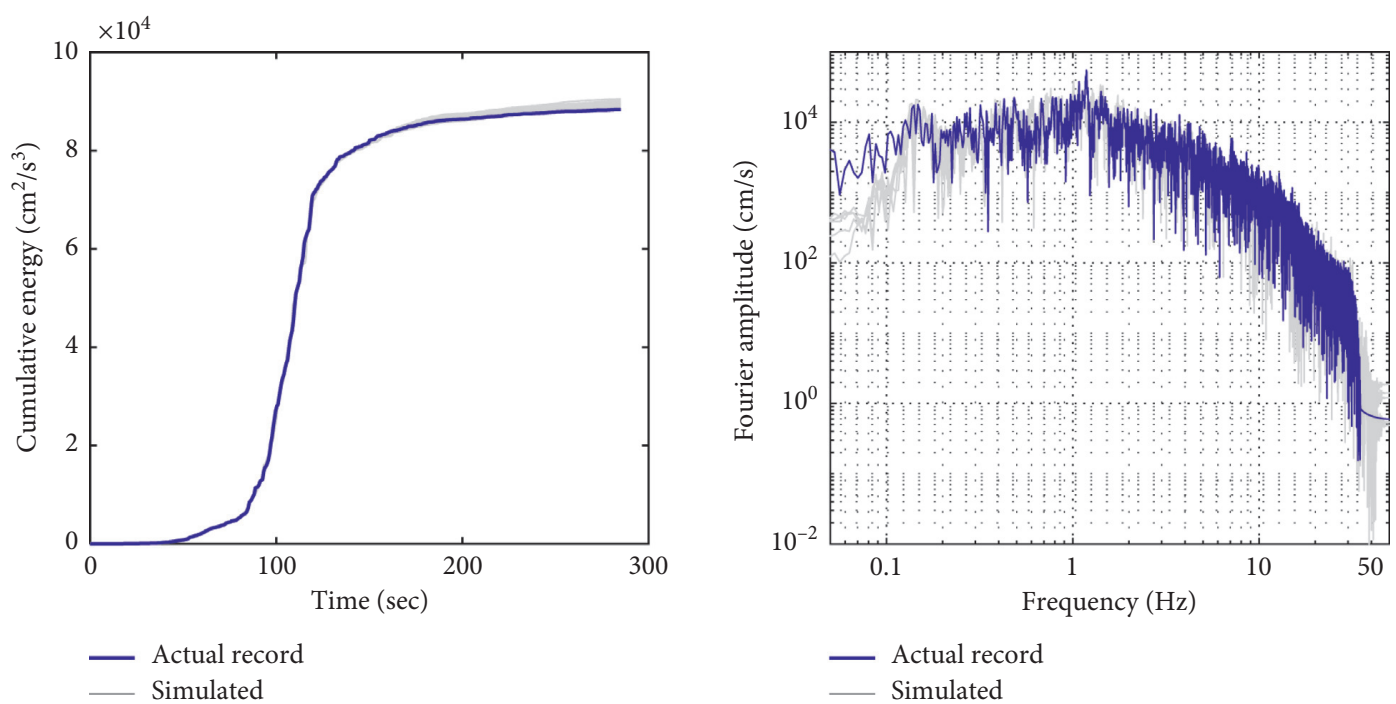

(b)

FiguRE 21: Comparison between four realizations and the recorded motions in terms of Fourier amplitudes and cumulative energy. (a) CHB002EW and (b) TKY023EW.
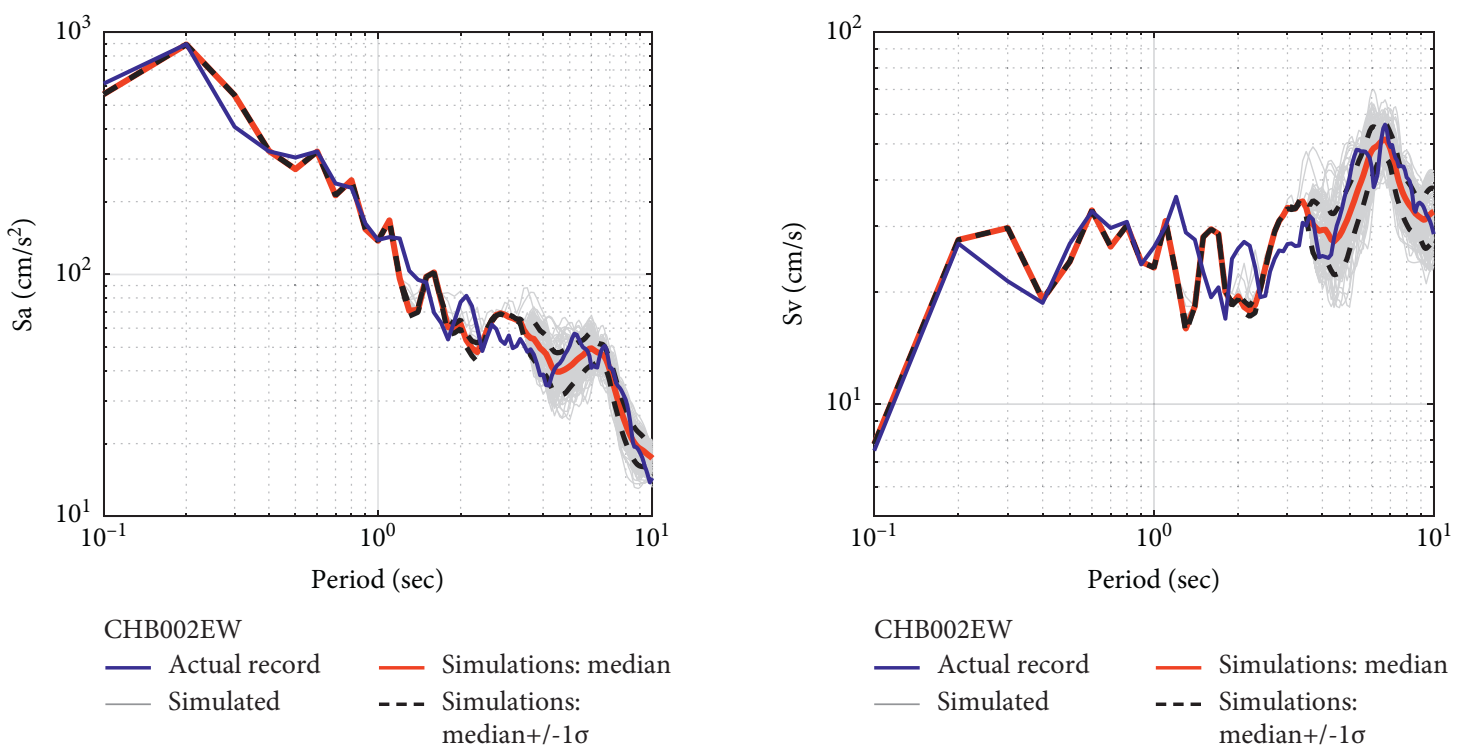

(a)

Figure 22: Continued. 


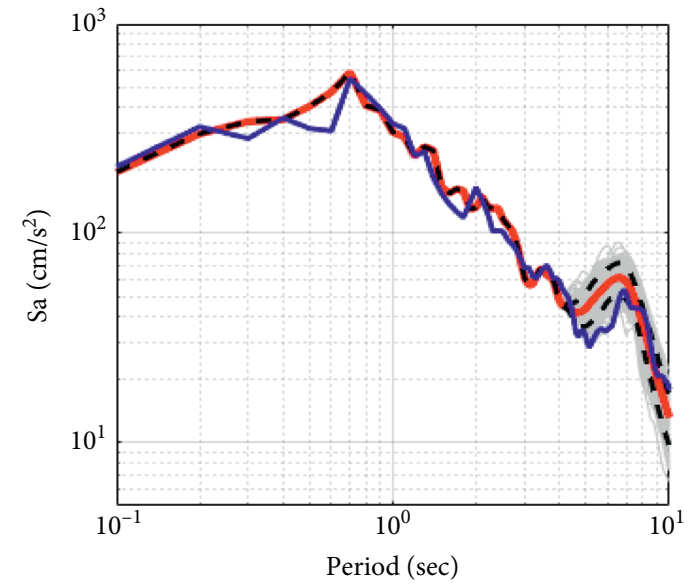

TKY023EW

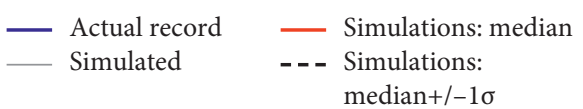

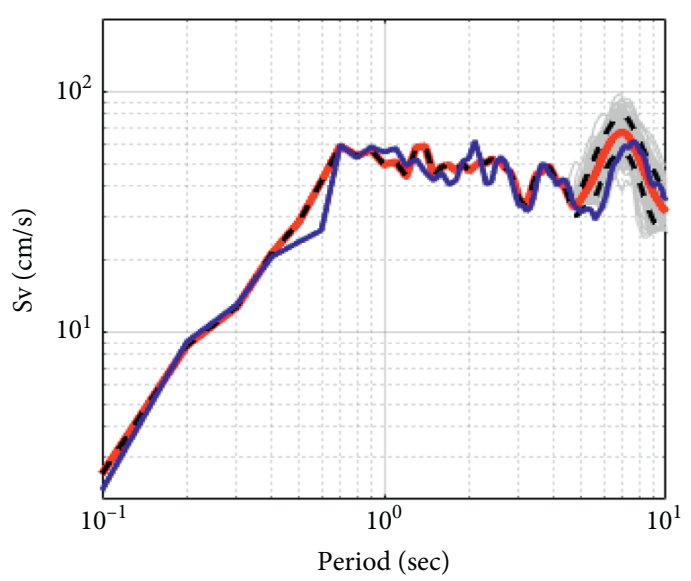

TKY023EW

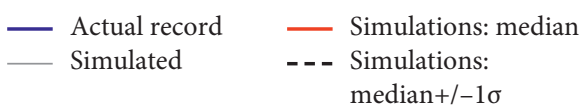

(b)

Figure 22: Response spectra (solid blue line) for the records CHB002EW and TKY023EW. Of 100 simulated long-period ground motions (thin gray lines), their median (thick red line) and median plus and minus one standard deviation $\pm \sigma$ (dashed lines) are involved; acceleration spectra on the left side; velocity spectra on the right side. (a) CHB002EW and (b) TKY023EW.

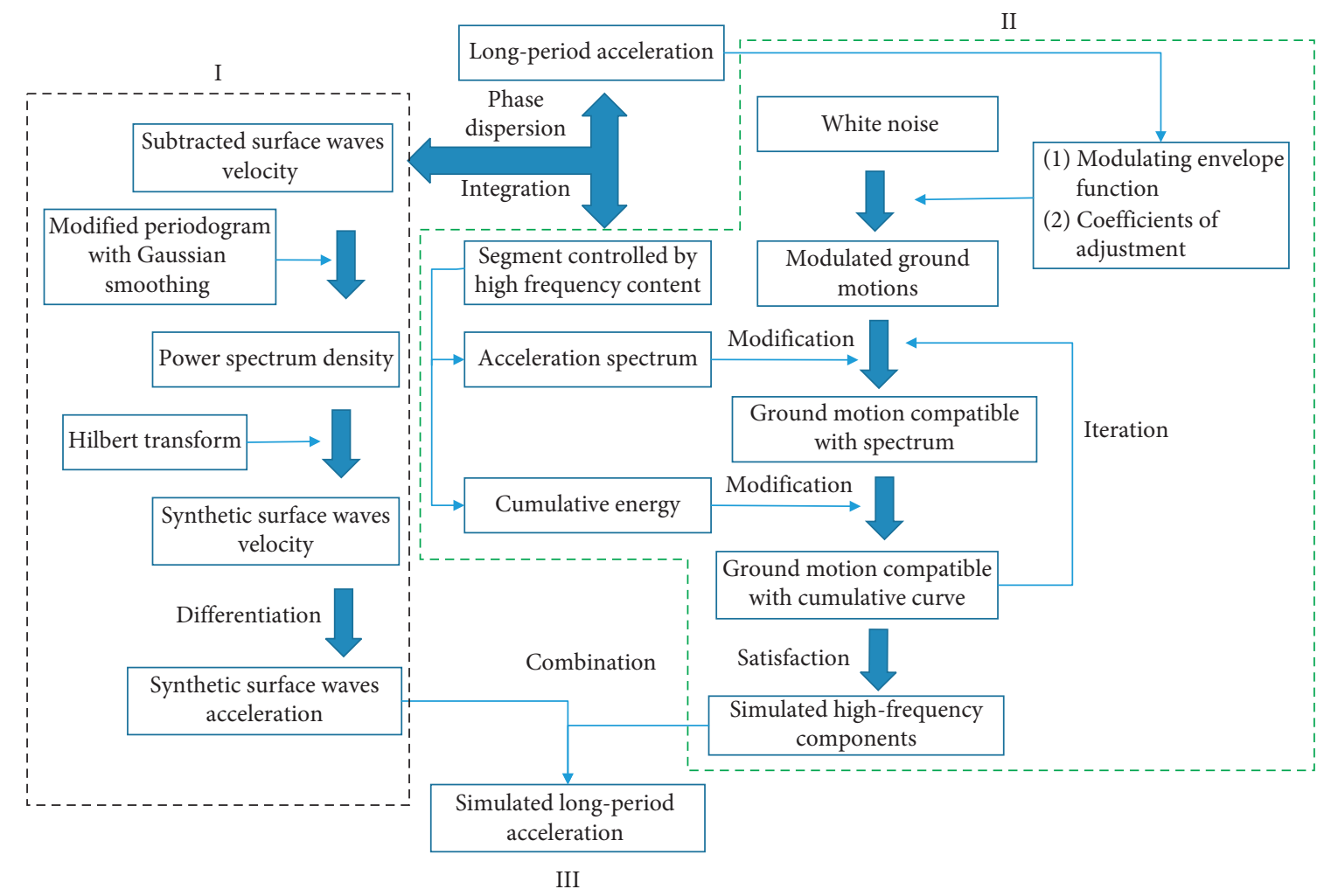

FIGURE 23: Schematic plot for generating long-period ground motions.

time series are obtained by multimodal point method [29]; because of these, the simulated LALP velocity surface waves are generated by means of spectral representation shown in Figure 24(d).
For the second phase, an initial seed motion is generated from the modification process, in which a Gaussian random process is modified in time domain by using a piecewise function and in frequency domain by using a set of fitted 


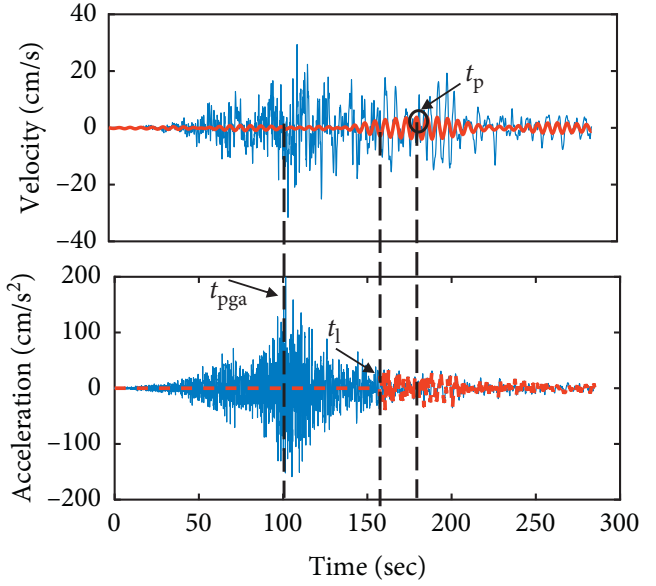

(a)

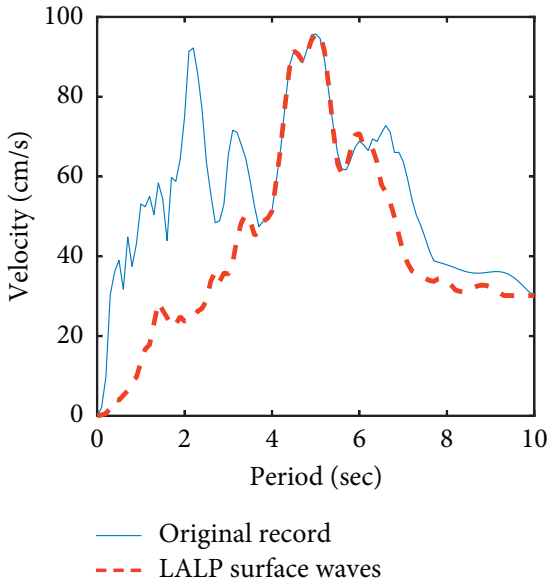

(b)

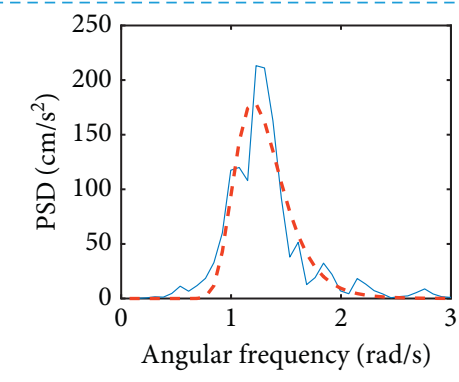

_ Actual record

- - - Fitted model

(c)

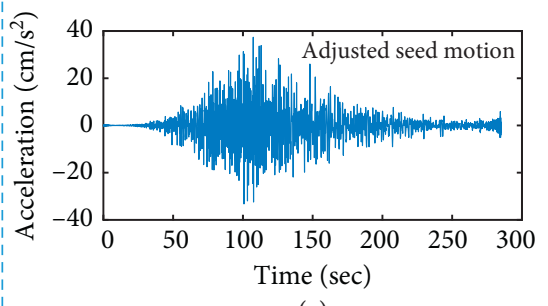

(e)

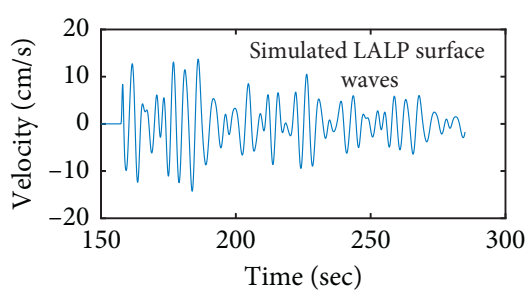

(d)

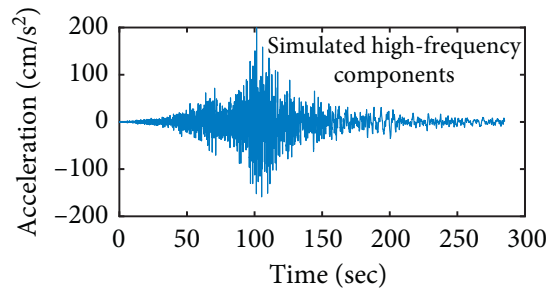

(f)

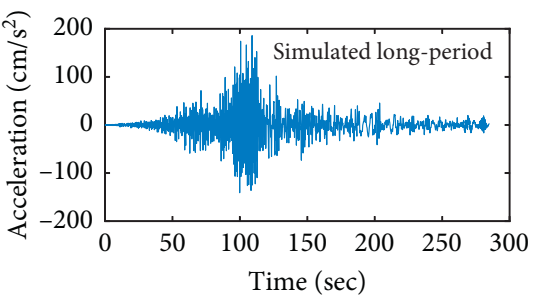

(g)

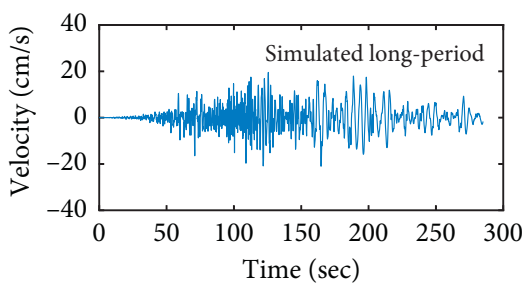

(h)

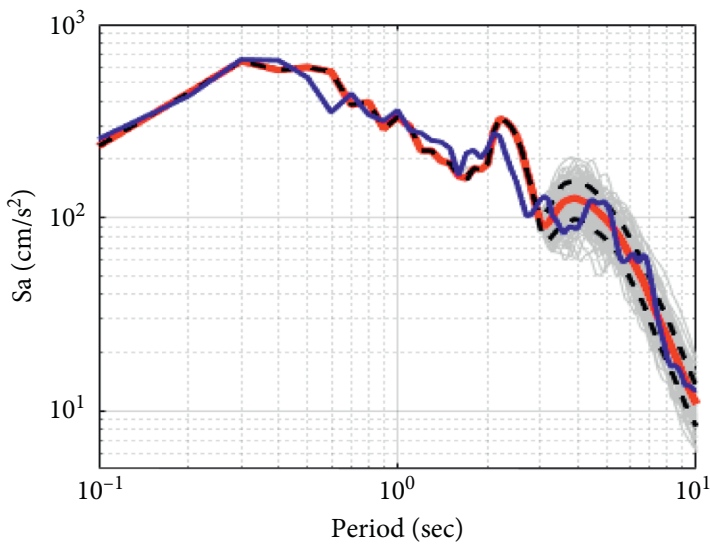

Actual record - Simulations: median Simulated - - - Simulations: median $\pm 1 \sigma$ (i)

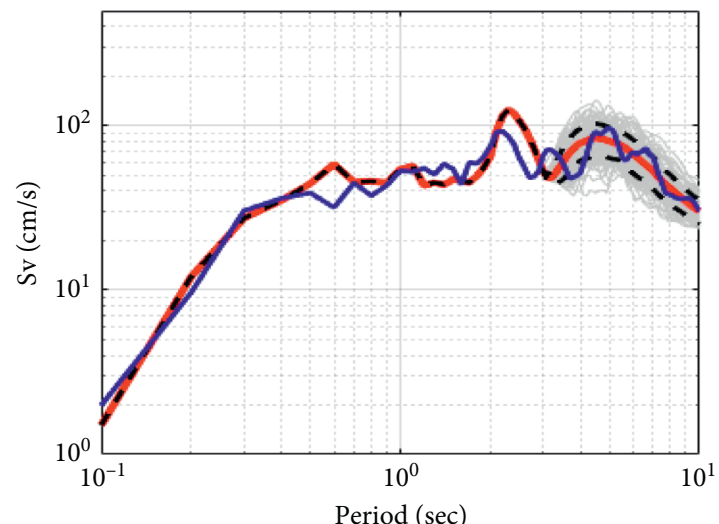

— Actual record - Simulations: median — Simulated - - - Simulations: median $\pm 1 \sigma$

(j)

FIgURE 24: Example to illustrate the proposed method. 
power spectrum functions; accordingly, the adjusted seed motion is presented in Figure 24(e); then, the iterative adjustments result in the high-frequency motion matching well with the target acceleration spectrum at short periods and cumulative energy curve, and the corresponding product is shown in Figure 24(f).

For the final phase, the LALP acceleration time series is attained from the differentiation of the resulting velocity series, and then it is superimposed into the high-frequency motion; consequently, one sample for the simulated longperiod motions by the proposed method is shown in the form of acceleration and velocity in Figures 24(g) and 24(h), respectively. Furthermore, 100 realizations are generated to verify the matching well to the target spectra, as illustrated in Figures 24(i) and 24(j).

It should be pointed out that the proposed method towards representative records is presented herein to demonstrate the feasibility for generating long-period motions. In addition, parameter values that describe stochastic property, LALP surface waves, and high-frequency components are determined individually for each record. Because of this, the methodology can be applied to other set of the motions with similar properties.

Finally, it is noted that the proposed method is suitable for simulation of long-period ground motions with narrowband long-period properties. This results from the analytical model for the power spectrum density. In addition, provided that the later-arriving long-period components are not capable of representing the gross long-period properties, the criterion for determination of long-period components may appear to be less effective.On the other hand, future studies appear to investigate a relationship between the identified parameters and seismic variables and extend the method to simulate long-period motion based on specific seismic environment, thus improving the engineering applicability.

\section{Data Availability}

The data used in the paper are available from the authors upon request.

\section{Conflicts of Interest}

The authors declare that they have no conflicts of interest.

\section{Acknowledgments}

The authors acknowledge support from the National Natural Science Foundation of China, with award number 51478068, and also express gratitude to National Research Institute for Earthquake Science and Disaster Prevention (K-NET and KiK-net), which offers access to dataset for studies.

\section{References}

[1] I. Okawa, T. Kashima, S. Koyama, M. Iiba, and M. Çelebi, "Summary of recorded building responses during the 2011 off the Pacific Coast of Tohoku earthquake with some implications to design motions," in Proceedings of the International
Symposium on Engineering Lessons Learned from the 2011 Great East Japan Earthquake, pp. 1049-1060, Tokyo, Japan, March 2012.

[2] K. Hatayama, "Lessons from the 2003 Tokachi-oki, Japan, earthquake for prediction of long-period strong ground motions and sloshing damage to oil storage tanks," Journal of Seismology, vol. 12, no. 2, pp. 255-263, 2008.

[3] T. C. Shin and T. L. Teng, "An overview of the 1999 Chi-Chi, Taiwan, earthquake," Bulletin of the Seismological Society of America, vol. 91, no. 5, pp. 895-913, 2001.

[4] I. Takewaki, S. Murakami, K. Fujita, S. Yoshitomi, and M. Tsuji, "The 2011 off the Pacific coast of Tohoku earthquake and response of high-rise buildings under long-period ground motions," Soil Dynamics and Earthquake Engineering, vol. 31, no. 11, pp. 1511-1528, 2011.

[5] N. Yamada and T. Iwata, "Long-period ground motion simulation in the Kinki area during the MJ 7.1 foreshock of the 2004 off the Kii peninsula earthquakes," Earth, Planets and Space, vol. 57, no. 3, pp. 197-202, 2005.

[6] G. Ahmadi, "Generation of artificial time-histories compatible with given response spectra-a review," Solid Mechanics Archives, vol. 4, no. 3, pp. 207-239, 1979.

[7] A. G. Dario and H. V. Erik, Simulated Earthquake Motions Compatible with Prescribed Response Spectra, Department of Civil Engineering: MIT, Cambridge, MA, USA, 1976.

[8] A. Giaralis and P. D. Spanos, "Wavelet-based response spectrum compatible synthesis of accelerograms-Eurocode application (EC8)," Soil Dynamics and Earthquake Engineering, vol. 29, no. 1, pp. 219-235, 2009.

[9] P. D. Spanos, A. Giaralis, and J. Li, "Synthesis of accelerograms compatible with the Chinese GB 50011-2001 design spectrum via harmonic wavelets: artificial and historic records," Earthquake Engineering and Engineering Vibration, vol. 8, no. 2, pp. 189-206, 2009.

[10] Y. Yamamoto and J. W. Baker, "Stochastic model for earthquake ground motion using wavelet packets," Bulletin of the Seismological Society of America, vol. 103, no. 6, pp. 3044-3056, 2013.

[11] D. Huang and G. Wang, "Energy-compatible and spectrumcompatible (ECSC) ground motion simulation using wavelet packets," Earthquake Engineering \& Structural Dynamics, vol. 46, no. 11, pp. 1855-1873, 2017.

[12] D. M. Boore, "Basin waves on a seafloor recording of the 1990 upland, California, earthquake: implications for ground motions from a larger earthquake," Bulletin of the Seismological Society of America, vol. 89, no. 1, pp. 317-324, 1999.

[13] D. M. Boore, "Phase derivatives and simulation of strong ground motions," Bulletin of the Seismological Society of America, vol. 93, no. 3, pp. 1132-1143, 2003.

[14] W. B. Joyner, "Strong motion from surface waves in deep sedimentary basins," Bulletin of the Seismological Society of America, vol. 90, pp. 687-693, 2000.

[15] G.-Q. Wang, G.-Q. Tang, D. M. Boore et al., "Surface waves in the western Taiwan coastal plain from an aftershock of the 1999 Chi-Chi, Taiwan, earthquake," Bulletin of the Seismological Society of America, vol. 96, no. 3, pp. 821-845, 2006.

[16] M. H. Dai, Y. M. Li, S. Y. Liu, and Y. F. Dong, "Identification of far-field long-period ground motions using phase derivatives," Advances in Civil Engineering, vol. 2019, Article ID 1065830, 20 pages, 2019.

[17] K. Koketsu and H. Miyake, "A seismological overview of longperiod ground motion," Journal of Seismology, vol. 12, no. 2, pp. 133-143, 2008. 
[18] A. Moustafa and I. Takewaki, "Deterministic and probabilistic representation of near-field pulse-like ground motion," Soil Dynamics and Earthquake Engineering, vol. 30, no. 5, pp. 412-422, 2010.

[19] Q. Fu and C. Menun, "Seismic-environment-based simulation of near-fault ground motions," in Proceedings of the 13th World Conference on Earthquake Engineering, Vancouver, Canada, November 2004.

[20] G. P. Mavroeidis and A. S. Papageorgiou, "A mathematical representation of near-fault ground motions," Bulletin of the Seismological Society of America, vol. 93, no. 3, pp. 1099-1131, 2003.

[21] C. Menun and Q. Fu, "An analytical model for near-fault ground motions and the response of SD of systems," in Proceedings of the 7th U.S. National Conference on Earthquake Engineering, Boston, MA, USA, October 2002.

[22] K. Hatayama, T. Kanno, and K. Kudo, "Control factors of spatial variation of long-period strong ground motions in the Yufutsu sedimentary basin, Hokkaido, during the mw 8.0 2003 Tokachi-oki, Japan, earthquake," Bulletin of the Seismological Society of America, vol. 97, no. 4, pp. 1308-1323, 2007.

[23] T. Kagawa, B. M. Zhao, K. Miyakoshi, and K. Irikura, "Modeling of 3D basin structures for seismic wave simulations based on available information on the target area: case study of the Osaka basin, Japan," Bulletin of the Seismological Society of America, vol. 94, no. 4, pp. 1353-1368, 2004.

[24] M. Dabaghi and A. Der Kiureghian, "Stochastic model for simulation of near-fault ground motions," Earthquake Engineering \& Structural Dynamics, vol. 46, no. 6, pp. 963-984, 2017.

[25] B. W. Dickinson and H. P. Gavin, "Parametric statistical generalization of uniform-hazard earthquake ground motions," Journal of Structural Engineering, vol. 137, no. 3, pp. 410-422, 2010.

[26] D. Yang and J. Zhou, "A stochastic model and synthesis for near-fault impulsive ground motions," Earthquake Engineering \& Structural Dynamics, vol. 44, no. 2, pp. 243-264, 2015.

[27] J. R. Abraham, C. G. Lai, and A. Papageorgiou, "Basin-effects observed during the 2012 Emilia earthquake sequence in Northern Italy," Soil Dynamics and Earthquake Engineering, vol. 78, pp. 230-242, 2015.

[28] V. A. Corchete, "A methodology for filtering and inversion of surface waves: an example of application to the lithospheric structure determination of the South Iberia," Tecnociencia, vol. 8, no. 1, pp. 91-112, 2006.

[29] G. Xu, P. Shi, and X. Zhou, "Artificial ground motion based on target response spectra and envelope," Earthquake Engineering and Engineering Dynamics, vol. 30, no. 1, pp. 1-7, 2010.

[30] Y. Li and G. Wang, "Simulation and generation of spectrumcompatible ground motions based on wavelet packet method," Soil Dynamics and Earthquake Engineering, vol. 87, pp. 44-51, 2016.

[31] I. Takewaki, K. Fujita, and S. Yoshitomi, "Uncertainties in long-period ground motion and its impact on building structural design: case study of the 2011 Tohoku (Japan) earthquake," Engineering Structures, vol. 49, pp. 119-134, 2013. 